hep-ph/0307058

FTUAM-03-12

IFT-UAM/CSIC-03-26

UCSD/PTH 03-08

\title{
Neutrino Physics in the Seesaw Model
}

\author{
A. Broncano ${ }^{\mathrm{a}, 1}$, M.B. Gavela ${ }^{\mathrm{a}, 2}$ and E. Jenkins ${ }^{\mathrm{b}, 3}$ \\ a Dept. de Física Teórica, C-XI, and IFT, C-XVI, Facultad de Ciencias, \\ Univ. Autónoma de Madrid, Cantoblanco, 28049 Madrid, Spain \\ b Dept. of Physics, University of California at San Diego, 9500 Gilman Drive, La Jolla, CA \\ 92093, USA
}

\begin{abstract}
The seesaw model of heavy and light Majorana neutrinos and its low-energy effective theory are studied, when the number of heavy neutrinos is equal to or less than the number of light lepton generations. We establish a general relationship between the high-energy parameters and the low-energy observables involving only the light fields. It is shown how low-energy measurements of the properties of light neutrinos suffice a priori to determine all couplings of the unobserved heavy neutrinos. $C P$ violation is present in low-energy processes if seesaw-model leptogenesis creates the matter-antimatter asymmetry of the universe.
\end{abstract}

\section{Introduction}

The leading high-energy theory of neutrino mass is the seesaw model [1], the minimal extension of the Standard Model (SM) which includes right-handed neutrino singlets with heavy Majorana masses. The seesaw model has a number of attractive features: (1) It explains the lightness of weakly-interacting neutrinos and the heaviness of sterile neutrinos via the seesaw mechanism [1. (2) It provides a natural solution for the generation of the matter-antimatter asymmetry of the universe. Leptogenesis occurs at high energies in heavy neutrino decay [2]. Approximately half of this lepton asymmetry is converted into a baryon asymmetry. (3) It is a renormalizable high-energy theory, so the number of high-energy parameters is finite. (4) It is natural: once right-handed neutrinos are added to the SM matter content, the Lagrangian containing all renormalizable terms allowed by $S U(3) \times S U(2)_{L} \times U(1)_{Y}$ gauge symmetry is the seesaw Lagrangian. (5) It can be embedded into a unified or partially unified theory

\footnotetext{
${ }^{1}$ alicia.broncano@uam.es

2 gavela@delta.ft.uam.es

3 ejenkins@ucsd.edu
} 
at a higher energy scale. The inclusion of right-handed neutrinos is natural in theories with extended gauge interactions at high energy, such as $S U(4) \times S U(2)_{L} \times S U(2)_{R}, S U(5) \times U(1)$, $S O(10)$ and $E 6$. In theories of this type, the asymmetry of the gauge interactions of lefthanded and right-handed fermions, as well as the asymmetry between the heavy and light Majorana neutrinos, is a consequence of the symmetry breakdown of the vacuum state rather than an asymmetry in the underlying gauge interactions. The Majorana mass term for the right-handed neutrinos is the order parameter for breaking the unified or partially unified gauge symmetry down to $S U(3) \times S U(2)_{L} \times U(1)_{Y}$.

An alternative theory for neutrino mass is obtained by making the ad hoc assumption that lepton number $L$ is exactly conserved. In this theory, neutrinos acquire only Dirac masses via the standard Higgs mechanism of electroweak symmetry breaking since $L$-violating Majorana neutrino masses are forbidden. The model of Dirac neutrino mass is disfavored relative to the seesaw model for the following reasons: (1) It fails to explain the mass hierarchy between neutrinos and other fermions. In order to account for experimental data, the Yukawa couplings of neutrinos must be many orders of magnitude smaller than those of all the other fermions. (2) The exact conservation of $L$, and consequently $(B-L)$ so long as baryon number $B$ is conserved, forbids generation of the baryon asymmetry at energy scales above the electroweak scale. (3) It is unnatural, since Majorana mass terms of right-handed neutrinos are not forbidden by the SM gauge symmetry.

Although very light neutrino masses become natural in the seesaw model, the electroweak hierarchy problem remains, since there are radiative corrections to the Higgs mass which are quadratic in the new heavy Majorana neutrino scale. As is well-known, supersymmetry could soften this electroweak fine-tuning problem. We concentrate, however, on the minimal (non-supersymmetric) seesaw model throughout this paper, in order to study the essential properties of the seesaw mechanism. Any beyond-the-SM theory, supersymmetric or not, which incorporates the seesaw mechanism, naturally is expected to include the general properties analyzed here.

In order to establish whether the seesaw model is the theory of nature, it is important to work out the low-energy implications of the seesaw theory. Our aim is to establish the connection of low-energy observables to the parameters of the underlying high-energy minimal seesaw model, without any ad hoc assumption on the values of the latter. In particular, it is very important to determine the connection of the $C P$-odd phases required for leptogenesis and a hypothetical future discovery of low-energy leptonic $C P$-violation. Our aim is to go farther than the usual general assertion that the low-energy phases are general combinations of all high-energy phases and moduli.

At low energies, there are two types of phases. Those contributing to $\Delta L=0$ transitions, for instance to neutrino-neutrino oscillations (such as the leptonic $C K M$-like phase $\delta$ in the case of three light neutrino generations [3]), are customarily called Dirac phases. Those contributing only to $\Delta L \neq 0$ transitions are designated Majorana phases [4]. Information on the latter could be extracted, in principle, from a positive measurement of neutrinoless double beta decay, combined with a determination of the absolute neutrino mass scale from tritium decay and with the observed values of the leptonic mixing angles. Even assuming that all those positive signals are indeed obtained in the future, a definite claim on the discovery of $C P$-violation from those data may be extremely difficult, if not impossible [5], although 
the subject is debatable [6]. On the contrary, the $C K M$-like leptonic phase $\delta$ may well be in reach in future superbeam facilities and/or neutrino factories [7, 8], provided the leptonic angle $\theta_{13}$ is not too small. A hypothetical measurement of $\delta$, together with the determination of the Majorana character of the neutrinos, would not be, by itself, a proof of leptogenesis as the origin of the matter-antimatter asymmetry of the universe, but it would make the argument for leptogenesis extremely compelling.

In Ref. [9], we constructed a low-energy effective theory for the seesaw model by integrating out the heavy Majorana neutrinos. The effective Lagrangian is of the form

$$
\mathcal{L}_{\text {eff }}=\mathcal{L}_{\mathrm{SM}}+\delta \mathcal{L}^{d=5}+\delta \mathcal{L}^{d=6}+\cdots,
$$

where $\mathcal{L}_{\mathrm{SM}}$ is the Standard Model Lagrangian and the higher-dimensional operators give the low-energy physics effects of the heavy Majorana neutrinos.

The leading higher-dimensional operator in the effective theory is the unique $d=5$ operator [10] which can be built with Standard Model fields:

$$
\delta \mathcal{L}^{d=5}=\frac{1}{2} c_{\alpha \beta}^{d=5}\left(\overline{\ell_{L \alpha}} \tilde{\phi}^{*}\right)\left(\tilde{\phi}^{\dagger} \ell_{L \beta}\right)+\text { h.c. }
$$

where $\ell_{L}$ are the lepton weak doublets and $\tilde{\phi}$ is related to the standard Higgs doublet by $\tilde{\phi}=i \tau_{2} \phi^{*}$. The $d=5$ operator coefficients are given in terms of the parameters of the high-energy seesaw theory by

$$
c^{d=5}=\left(Y_{\nu}^{*}\left(M^{*}\right)^{-1} Y_{\nu}^{\dagger}\right)
$$

where $M$ denotes the heavy neutrino mass matrix, and $Y_{\nu}$ is the Yukawa coupling matrix between the heavy neutrinos and the charged lepton doublets.

When the electroweak gauge symmetry breaks spontaneously, this $d=5$ operator yields a $d=3$ Majorana mass term for the left-handed weakly interacting neutrinos. Rewriting the theory in terms of the Majorana mass eigenstate neutrinos leads to the presence of a non-trivial lepton mixing matrix in the couplings of $W$ bosons to lepton charged currents.

In Ref. [9], the $d=6$ term of the low-energy effective theory was determined to be

$$
\delta \mathcal{L}^{d=6}=c_{\alpha \beta}^{d=6}\left(\overline{\ell_{L \alpha}} \tilde{\phi}\right) i \not \partial\left(\tilde{\phi}^{\dagger} \ell_{L \beta}\right)
$$

where the $d=6$ operator coefficients are given in terms of the parameters of the high-energy seesaw theory by

$$
c^{d=6}=Y_{\nu}\left(|M|^{2}\right)^{-1} Y_{\nu}^{\dagger} .
$$

When the Higgs doublet acquires a vacuum expectation value, this $d=6$ operator leads to $d=4$ kinetic energy terms for the left-handed Majorana neutrinos. Other $d=6$ operators will be present in the low-energy Lagrangian, since they are generated by radiative mixing of the above operator in the renormalization group running between the high-energy and low-energy scales. These other $d=6$ operators will not be discussed in this work, as their effects are subdominant [11].

In Ref. 9], we showed that the low-energy effective theory including only the $d=5$ and $d=6$ operators contains an equal (a greater) number of real and imaginary parameters as the 
high-energy seesaw model when the number of right-handed neutrinos in the seesaw theory is equal to (less than) the number of generations of Standard Model fermions. Thus, low-energy measurement of the $d=5$ and $d=6$ operator coefficients suffices a priori to determine all of the parameters of the high-energy seesaw theory.

While the direct physical effects of the $d=5$ operator, such as the light neutrino masses, are proportional to $1 / M$, those of the $d=6$ operator are suppressed by $1 / M^{2}$ and their experimental measurement is presently out of reach, at least for natural values of the heavy seesaw scale. Nevertheless, the ensemble of the $d=5$ and $d=6$ operators is the lowenergy tell-tale of the seesaw mechanism for any theory beyond the SM which includes that mechanism, barring most unnatural cancellations, and it deserves particular study. In this work, we make explicit the connection of the high-energy parameters of the seesaw model to the $d=5$ and $d=6$ operator coefficients. We will see that the analysis of this operator structure sheds light on the relation between the seesaw model and planned experiments.

We work out the general relation between the high-energy seesaw parameters in $Y_{\nu}$ and $M$ and the $d=5$ and $d=6$ operator coefficients of the low-energy effective theory, for an arbitrary number of light generations. This analysis allows us to express the combination of high-energy phases contributing to leptogenesis in terms of only low-energy parameters in a very compact form. The most important physical consequences will be already transparent from the general formulae, which are basis-independent.

For detailed computations, it is necessary to specify a basis. A standard high-energy basis for the seesaw model is defined. No unphysical parameters occur in the standard high-energy basis; for this reason, it is the simplest choice of basis. Besides including the customary choice of diagonal $M$ and $Y_{e}$, the standard high-energy basis expresses the seesaw theory in terms of physical, basis-independent, moduli and phases of $Y_{\nu}$. For the case of two generations, $Y_{\nu}$ contains 2 high-energy phases, whereas for three generations, it contains 6 phases. The connection between the standard high-energy basis and the low-energy bases is made.

The lessons learned in the general case will be illustrated in full detail for the minimal seesaw model with two heavy and light generations, as an important special case. The generalization to three generations is straightforward, but more complicated, and will be published in a separate work. Leptogenesis already occurs for two generations of leptons since there are $C P$-odd phases present in the lepton sector due to the Majorana character of neutrinos. Thus, interesting $C P$-violating effects occur in low-energy processes with only two generations of leptons, and can be studied in this simpler context. The low-energy effective theory including the $d=5$ operator contains only a single $C P$-odd phase in the lepton mixing matrix, while there are two independent phases at high energies. Thus, not all of the parameters in the seesaw model appear in the low-energy effective theory with only the $d=5$ operator. Indeed, the $d=6$ operator coefficient contains an additional $C P$-violating phase which is independent of the phase of the lepton mixing matrix. It is very instructive to work out in detail the connection between leptogenesis and the two low-energy $C P$-odd phases.

The organization of the paper is as follows. Section 2 reviews the high-energy seesaw Lagrangian. The standard high-energy basis for the seesaw model is defined, and the leptogenesis asymmetry is discussed. Section 3 presents the $d \leq 6$-effective Lagrangian in the low-energy spontaneously-broken theory. Physical observables and global symmetries of the effective Lagrangian are discussed as well. Section 4 gives the general connection between the 
high-energy parameters of the seesaw model and the low-energy parameters of the effective theory, and relates high-energy and low-energy observables. The relationship of our results with those in the literature also is discussed. Section 5 specializes the previous general results to the seesaw model with two generations of heavy neutrinos and light leptons. Additional results are provided in the appendices. Appendix 1 considers the relationship of our standard high-energy basis to alternative high-energy bases with additional unphysical parameters, for any number of lepton generations. Appendices 2, 3 and 4 concern the case of two generations: they contain, respectively, formulae relating the flavor and mass eigenstate bases of the light neutrinos, the generalizations of the low-energy observables to include the effects of the $d=6$ operator, and the determination of the heavy Majorana neutrino masses from low-energy observables. Appendix 5 contains the Feynman rules for neutrinos at high and low energies.

\section{Seesaw Lagrangian}

The seesaw Lagrangian is the most general renormalizable Lagrangian which can be written for the Standard Model gauge group with right-handed neutrinos included in the fermion content of the theory. The leptonic Lagrangian of the seesaw model is given by:

$$
\mathcal{L}_{\text {leptons }}=\mathcal{L}_{\text {leptons }}^{\mathrm{KE}}+\mathcal{L}_{\text {leptons }}^{\chi \mathrm{SB}}
$$

where

$$
\mathcal{L}_{\text {leptons }}^{\mathrm{KE}}=i \overline{\ell_{L}} \not D \ell_{L}+i \overline{e_{R}} \not D e_{R}+i \overline{N_{R}} \not \partial N_{R}
$$

contains the kinetic energy and gauge interaction terms of the left-handed lepton doublets $\ell_{L}$, the right-handed charged leptons $e_{R}$, and the right-handed neutrinos $N_{R}$, and

$$
\mathcal{L}_{\text {leptons }}^{\chi \mathrm{SB}}=-\overline{\ell_{L}} \phi Y_{e} e_{R}-\overline{\ell_{L}} \widetilde{\phi} Y_{\nu} N_{R}-\frac{1}{2} \overline{N_{R}{ }^{c}} M N_{R}+\text { h.c. },
$$

contains the Yukawa interactions and the Majorana mass term of the gauge-singlet righthanded neutrinos. The kinetic energy Lagrangian of the lepton sector is invariant under the chiral transformations

$$
\ell_{L} \rightarrow V_{\ell} \ell_{L}, \quad e_{R} \rightarrow V_{e} e_{R}, \quad N_{R} \rightarrow V_{N} N_{R}
$$

where $V_{\ell}$ and $V_{e}$ are $n \times n$ unitary matrices for $n$ generations of light leptons, and $V_{N}$ is an $n^{\prime} \times n^{\prime}$ unitary matrix for $n^{\prime}$ generations of right-handed neutrinos. The chiral symmetry $G=U(n)_{\ell} \times U(n)_{e} \times U\left(n^{\prime}\right)_{N}$ of the lepton kinetic energy terms is completely broken by the chiral symmetry-breaking Lagrangian $\mathcal{L}^{\chi \mathrm{SB}} \cdot \mathcal{L}^{\chi \mathrm{SB}}$ would respect the chiral symmetry if the matrices $Y_{e}, Y_{\nu}$ and $M$ transformed as

$$
\begin{aligned}
Y_{e} & \rightarrow V_{\ell} Y_{e} V_{e}^{\dagger}, \\
Y_{\nu} & \rightarrow V_{\ell} Y_{\nu} V_{N}^{\dagger}, \\
M & \rightarrow V_{N}^{*} M V_{N}^{\dagger},
\end{aligned}
$$

under the chiral symmetry. Eq. (10) implies that seesaw theories with Yukawa and Majorana mass matrices related by chiral transformations are equivalent theories. Thus, it is possible 
to use the chiral transformations to write the explicit chiral symmetry-breaking parameters in a convenient basis. As shown in Ref. [9], the number of physical parameters in $Y_{e}, Y_{\nu}$ and $M$ is equal to the number of parameters in $Y_{e}, Y_{\nu}$ and $M$ minus the number of parameters in $V_{\ell}, V_{e}$ and $V_{N}$. The seesaw model contains $\left(n+n^{\prime}+n n^{\prime}\right)$ real and $n\left(n^{\prime}-1\right)$ imaginary physical parameters in the matrices $Y_{e}, Y_{\nu}$, and $M$.

Let us use the chiral transformations of the high-energy seesaw model to define a standard high-energy basis which does not contain any spurious unphysical parameters. It will include the convenient and customary specialization to a basis in which both $Y_{e}$ and $M$ are diagonal and real.

In a general basis, the complex $n \times n$ matrix $Y_{e}$ depends on $n^{2}$ real and $n^{2}$ imaginary parameters. Let us denote by $y_{\alpha}$ the eigenvalues of $Y_{e}$, with $\alpha$ being the flavor index which, in a redundant notation, takes the values $\alpha=1,2, \cdots, n=e, \mu, \cdots, e_{n}$. The change of basis

$$
Y_{e} \rightarrow V_{\ell} Y_{e} V_{e}^{\dagger}=\left(Y_{e}\right)_{\text {diag, real }}=\operatorname{diag}\left(y_{e}, y_{\mu}, \cdots, y_{e_{n}}\right)
$$

determines $n(n-1)$ real and $n^{2}$ imaginary parameters of the matrices $V_{e}$ and $V_{\ell}$, which each contain $n(n-1) / 2$ real and $n(n+1) / 2$ imaginary parameters. All of the real and $n^{2}$ of the $n(n+1)$ imaginary parameters in $V_{e}$ and $V_{\ell}$ are determined by diagonalization of $Y_{e} ; n$ imaginary parameters in $V_{e}$ and $V_{\ell}$ are not determined since $\left(Y_{e}\right)_{\text {diag, real }}$ is left invariant by a transformation with

$$
V_{\ell}=V_{e}=\operatorname{diag}\left(e^{i \zeta_{1}}, e^{i \zeta_{2}}, \cdots, e^{i \zeta_{n}}\right),
$$

which depends on $n$ arbitrary phases $\zeta_{\alpha}, \alpha=1, \cdots, n$.

In a general basis, the complex symmetric $n^{\prime} \times n^{\prime}$ matrix $M$ contains $n^{\prime}\left(n^{\prime}+1\right) / 2$ real and $n^{\prime}\left(n^{\prime}+1\right) / 2$ imaginary parameters. The change of basis

$$
M \rightarrow V_{N}^{*} M V_{N}^{\dagger}=M_{\text {diag, real }}=\operatorname{diag}\left(\left|M_{1}\right|,\left|M_{2}\right|, \cdots,\left|M_{n^{\prime}}\right|\right),
$$

fully determines the $n^{\prime}\left(n^{\prime}-1\right) / 2$ real and $n^{\prime}\left(n^{\prime}+1\right) / 2$ imaginary parameters of the $n^{\prime} \times n^{\prime}$ unitary matrix $V_{N}$. $M_{\text {diag, real }}$ depends on the $n^{\prime}$ real mass eigenvalues of the heavy neutrinos. No further transformation by $V_{N}$ is allowed. In this basis, the heavy Majorana mass eigenstate neutrinos are

$$
N_{i}=N_{R i}+N_{R i}^{c}
$$

and satisfy the condition $N_{i}^{c}=N_{i}$.

The leptonic Lagrangian of the seesaw model, expressed in terms of the heavy Majorana mass eigenstate neutrinos $N_{i}$, is then given by Eq. (6) with

$$
\begin{aligned}
& \mathcal{L}_{\text {leptons }}^{\mathrm{KE}}=i \overline{\ell_{L}} \not D \ell_{L}+i \overline{e_{R}} \not D e_{R}+\frac{1}{2} i \bar{N}_{i} \not \partial N_{i} \\
& \mathcal{L}_{\text {leptons }}^{\chi \mathrm{SB}}=-\overline{\ell_{L}} \phi Y_{e} e_{R}-\frac{1}{2} \overline{\ell_{L}} \widetilde{\phi} Y_{\nu} N_{i}-\frac{1}{2} \bar{N}_{i}\left|M_{i}\right| N_{i}+\text { h.c. }
\end{aligned}
$$

In the basis in which $Y_{e}$ and $M$ are both diagonal and real, the complex $n \times n^{\prime}$ matrix $Y_{\nu}$ contains $n n^{\prime}$ real and $n n^{\prime}$ imaginary parameters, which are the magnitudes and phases of the $Y_{\nu}$ matrix elements

$$
\left(Y_{\nu}\right)_{\alpha j} \equiv u_{\alpha j} e^{i \phi_{\alpha j}}
$$


where $j=1, \cdots, n^{\prime}$ is the heavy Majorana neutrino mass eigenstate index.

Under a $V_{\ell}=V_{e}$ rephasing, $\phi_{\alpha j} \rightarrow \phi_{\alpha j}+\zeta_{\alpha}$. To specify the phases $\zeta_{\alpha}$ is to choose a basis. The $n n^{\prime}$ phases in the matrix $Y_{\nu}$ consist of $n\left(n^{\prime}-1\right)$ independent phases,

$$
\Phi_{\alpha j} \equiv\left(\phi_{\alpha j}-\Psi_{\alpha}\right)=\phi_{\alpha j}-\frac{1}{n^{\prime}} \sum_{k=1}^{n^{\prime}} \phi_{\alpha k},
$$

which are invariant under $\zeta_{\alpha}$ rephasings, and $n$ phases,

$$
\Psi_{\alpha} \equiv \frac{1}{n^{\prime}} \sum_{j=1}^{n^{\prime}} \phi_{\alpha j}
$$

which transform under $\zeta_{\alpha}$ rephasings as $\Psi_{\alpha} \rightarrow \Psi_{\alpha}+\zeta_{\alpha}$. The basis-dependent phases $\Psi_{\alpha}$ are unphysical, and can be removed from $Y_{\nu}$ by fixing

$$
\zeta_{\alpha}=-\Psi_{\alpha}
$$

The phases in each row of $Y_{\nu}$ then satisfy the constraint

$$
\sum_{j=1}^{n^{\prime}} \Phi_{\alpha j}=0
$$

In summary, for this choice of basis, the $Y_{\nu}$ matrix elements

$$
\left(Y_{\nu}\right)_{\alpha j}=u_{\alpha j} e^{i \Phi_{\alpha j}}
$$

depend on only $n\left(n^{\prime}-1\right)$ physical high-energy phases $\Phi_{\alpha j}, \alpha \neq j$, since the phases $\Phi_{\alpha j}$, $\alpha=j$, are not independent and are determined in terms of these phases using Eq. (21). of the $n\left(n^{\prime}-1\right)$

The basis with $Y_{e}$ and $M$ both diagonal and real, and with $Y_{\nu}$ given by Eqs. (21) and (22) will be called the standard high-energy basis. In this basis, the $\zeta_{\alpha}$ are chosen to be independent of the $n\left(n^{\prime}-1\right)$ physical phases, which is the simplest choice. Alternative, more complicated, bases are possible. One class of these bases is presented in Appendix 1. Another class includes the physical low-energy basis, for which the $\zeta_{\alpha}$ have a dependence on the physical high-energy phases. This low-energy basis will be described later in the paper in subsection 3.3 .

In general, a given high-energy theory is described initially by arbitrary matrices $M, Y_{\nu}$ and $Y_{e}$. When rewriting the theory in the standard high-energy basis, all physical phases, including those stemming from the Majorana character of the heavy neutrinos contained initially in $M$, are transferred to the matrix $Y_{\nu}$ by the diagonalization of $M$ and $Y_{e}$.

Leptogenesis occurs at high energy in the seesaw model due to the presence of $C P$ violation in the decays of the heavy Majorana neutrinos $N_{i}$ to light leptons and Higgs bosons [2, 12]. The $C P$ asymmetry produced in the decay of $N_{i}$ is given by

$$
\epsilon_{i}=\frac{1}{\pi\left(Y_{\nu}^{\dagger} Y_{\nu}\right)_{i i}} \sum_{j \neq i}^{n^{\prime}}\left\{\operatorname{Im}\left[\left(Y_{\nu}^{\dagger} Y_{\nu}\right)_{i j}\right]^{2} f\left(\frac{\left|M_{j}\right|^{2}}{\left|M_{i}\right|^{2}}\right)\right\}
$$


where $f$ is the loop function [2, 12, 13.

$$
f(x)=\sqrt{x}\left[\frac{2-x}{1-x}-(1+x) \ln \left(\frac{1+x}{x}\right)\right] .
$$

Notice that the $C P$-asymmetries are invariant under $V_{\ell}$ transformations, since both $M$ and the matrix elements

$$
\left(Y_{\nu}^{\dagger} Y_{\nu}\right)_{i j}=\sum_{\alpha=1}^{n} u_{\alpha i} u_{\alpha j} e^{i\left(\Phi_{\alpha j}-\Phi_{\alpha i}\right)}
$$

are invariant under these transformations. Thus, the lepto-asymmetries are independent of the low-energy basis.

For non-degenerate heavy Majorana neutrinos, the lepton asymmetry is generated by decay of the lightest heavy Majorana neutrino $N_{1}$, and is proportional to the $C P$-asymmetry $\epsilon_{1}$. In terms of the physical high-energy parameters of the standard basis,

$$
\epsilon_{1}=\frac{1}{\pi\left(\sum_{\alpha} u_{\alpha 1}^{2}\right)} \sum_{j \neq 1}^{n^{\prime}}\left\{\operatorname{Im}\left[\left(\sum_{\alpha=1}^{n} u_{\alpha 1} u_{\alpha j} e^{i\left(\Phi_{\alpha j}-\Phi_{\alpha 1}\right)}\right)^{2}\right] f\left(\frac{\left|M_{j}\right|^{2}}{\left|M_{1}\right|^{2}}\right)\right\} \text {. }
$$

If leptogenesis in the seesaw model produces the matter-antimatter asymmetry of the universe, then the parameter $\epsilon_{1}$ is constrained by the experimental value of the baryon-tophoton ratio [14,

$$
\eta_{B}=\left(6.1_{-0.2}^{+0.3}\right) \times 10^{-10},
$$

which is related to the lepto-asymmetry by [15]

$$
\eta_{B} \simeq 0.01 \epsilon_{1} \cdot \kappa,
$$

where the factor $\kappa$ describes the washout of the produced lepton asymmetry due to lepton number-violating processes. For $M_{1} \ll M_{2}, M_{3}, \cdots, M_{n^{\prime}}$, the value of $\eta_{B}$ required by experiment typically is obtained when $\epsilon_{1} \simeq\left(10^{-6}-10^{-7}\right)$ and $\kappa \simeq\left(10^{-3}-10^{-2}\right)$. It is possible to obtain a lower bound on $M_{1}$ from the experimental value of $\eta_{B}$ and neutrino oscillation data [15],

$$
M_{1}>4 \times 10^{8} \mathrm{GeV} .
$$

This bound indicates a seesaw scale much higher than the scale of electroweak symmetry breaking, and gives an estimate for the magnitude of the $1 / M$ suppression factor of the $d=6$ operator relative to the $d=5$ operator at low energies, provided seesaw leptogenesis generates the matter-antimatter asymmetry of the universe.

\section{Low-energy Effective Lagrangian}

After spontaneous symmetry breaking of the electroweak gauge symmetry, the effective Lagrangian for leptons is given in the flavor eigenstate basis by

$$
\begin{aligned}
\left(\mathcal{L}_{e f f}\right)_{\text {leptons }}= & i \bar{e}_{L \alpha} \not D e_{L \alpha}+i \bar{e}_{R \alpha} \not D e_{R \alpha}-m_{e_{\alpha}}\left(\bar{e}_{L} e_{R \alpha}+\bar{e}_{R} e_{L \alpha}\right)+\mathcal{L}_{\text {neutrino }} \\
& +\mathcal{L}_{\mathrm{W}, \mathrm{Z}}+\mathcal{L}_{\text {Higgs }}
\end{aligned}
$$


where the covariant derivative of the charged leptons contains the electromagnetic gauge field; the charged lepton mass matrix $\left(m_{e}\right)=\frac{v}{\sqrt{2}}\left(Y_{e}\right)$ is diagonal with real eigenvalues $m_{e_{\alpha}}$ in the flavor basis; $\mathcal{L}_{\text {neutrino }}$ (defined below) contains the kinetic energy and Majorana mass terms of the left-handed weakly-interacting neutrinos;

$$
\mathcal{L}_{\mathrm{W}, \mathrm{Z}}=\frac{g}{\sqrt{2}}\left(J_{\mu}^{-C C} W^{+\mu}+J_{\mu}^{+C C} W^{-\mu}\right)+\frac{g}{\cos \theta_{W}} J_{\mu}^{N C} Z^{\mu}
$$

contains the $W$ and $Z$ couplings to the weak charged and neutral currents, and $\mathcal{L}_{\text {Higgs }}$ contains the lepton couplings to the Higgs boson.

In the flavor basis, the charged and neutral currents are given by

$$
\begin{aligned}
J_{\mu}^{-C C} & =\bar{e}_{L \alpha} \gamma_{\mu} \nu_{L \alpha}, \\
J_{\mu}^{N C} & =\frac{1}{2} \bar{\nu}_{L \alpha} \gamma_{\mu} \nu_{L \alpha}+\left(-\frac{1}{2}-s^{2} \theta_{W}\right) \bar{e}_{L \alpha} \gamma_{\mu} e_{L \alpha}+\left(-s^{2} \theta_{W}\right) \bar{e}_{R \alpha} \gamma_{\mu} e_{R \alpha} .
\end{aligned}
$$

The neutrino couplings to the Higgs in $\mathcal{L}_{\text {Higgs }}$ depend on the higher-dimension $d>4$ operators of the effective theory.

\subsection{Low-energy $d \leq 5$-Effective Lagrangian}

The neutrino Lagrangian for the effective theory including only the $d=5$ operator of Eq. (2) is given in the flavor basis by

$$
\mathcal{L}_{\text {neutrino }}^{d \leq 5}=i \bar{\nu}_{L \alpha} \not \partial \nu_{L \alpha}-\frac{1}{2} \overline{\nu_{L}{ }^{c}}{ }_{\alpha} m_{\alpha \beta} \nu_{L \beta}-\frac{1}{2}{\overline{\nu_{L}}}_{\alpha} m_{\alpha \beta}^{*} \nu_{L \beta}{ }^{c},
$$

where the light neutrino Majorana mass matrix is [10] defined by

$$
m \equiv-\frac{v^{2}}{2} c^{d=5}
$$

The matrix $m$ is diagonalized by the transformation

$$
m \rightarrow V^{*} m V^{\dagger}=m_{\text {diag,real }}=\operatorname{diag}\left(m_{1}, m_{2}, \cdots, m_{n}\right),
$$

where the $n \times n$ unitary matrix $V$ contains $n(n-1) / 2$ real and $n(n+1) / 2$ imaginary parameters, and $m_{i}$ denote the real positive eigenvalues of the light Majorana neutrinos. The light neutrino Majorana mass eigenstates are defined by

$$
\nu_{i}=V_{i \alpha} \nu_{\alpha L}+V_{i \alpha}^{*} \nu_{\alpha L}^{c}
$$

and satisfy $\nu_{i}^{c}=\nu_{i}$. The leptonic Lagrangian of the $d \leq 5$ effective theory can be rewritten in terms of the light Majorana neutrino mass eigenstates,

$$
\mathcal{L}_{\text {neutrino }}^{d \leq 5}=\frac{1}{2} \bar{\nu}_{i}\left(i \overrightarrow{\not \supset}-m_{i}\right) \nu_{i}
$$

The weak currents also can be rewritten as

$$
J_{\mu}^{-C C}=\bar{e}_{L \alpha} \gamma_{\mu}\left(V^{\dagger}\right)_{\alpha i} \nu_{i}, \quad\left(J_{\mu}^{N C}\right)_{\text {neutrino }}=\frac{1}{2} \bar{\nu}_{i} \gamma_{\mu} \nu_{i}
$$


where $V$ now appears in the leptonic charged current. It is customary to reabsorb $n$ phases $\omega_{\alpha}$ of $V$ into the the charged lepton fields, $e^{i \omega_{\alpha}} e_{\alpha} \rightarrow e_{\alpha}$. The charged current then becomes

$$
J_{\mu}^{-C C}=\bar{e}_{L \alpha} \gamma_{\mu} U_{\alpha i} \nu_{i}
$$

where the lepton mixing matrix

$$
U \equiv \Omega V^{\dagger}, \Omega=\operatorname{diag}\left(e^{i \omega_{1}}, \ldots . ., e^{i \omega_{n}}\right),
$$

is the PMNS matrix 16, which can be written in the form

$$
U=U_{C K M} D_{M a j}
$$

where $U_{C K M}$ is the leptonic analogue of the $C K M$ matrix of the quark sector with $(n-2)(n-$ $1) / 2$ Dirac phases and $D_{M a j}$ is a diagonal matrix of $(n-1)$ Majorana phase differences. For two lepton generations,

$$
U=\left(\begin{array}{cc}
\cos \theta & \sin \theta \\
-\sin \theta & \cos \theta
\end{array}\right)\left(\begin{array}{cc}
e^{i \phi / 2} & 0 \\
0 & 1
\end{array}\right)
$$

contains a single Majorana phase difference $\phi$ and no $C K M$ phase. For three lepton generations,

$$
U=U_{C K M}\left(\begin{array}{ccc}
e^{i \phi_{1} / 2} & 0 & 0 \\
0 & e^{i \phi_{2} / 2} & 0 \\
0 & 0 & 1
\end{array}\right)
$$

contains two Majorana phase differences $\phi_{1}$ and $\phi_{2}$ in $D_{M a j}$, and a single $C K M$ phase $\delta$ in

$$
U_{C K M}=\left(\begin{array}{ccc}
c_{12} c_{13} & s_{12} c_{13} & s_{13} e^{-i \delta} \\
-s_{12} c_{23}-c_{12} s_{23} s_{13} e^{i \delta} & c_{12} c_{23}-s_{12} s_{23} s_{13} e^{i \delta} & s_{23} c_{13} \\
s_{12} s_{23}-c_{12} c_{23} s_{13} e^{i \delta} & -c_{12} s_{23}-s_{12} c_{23} s_{13} e^{i \delta} & c_{23} c_{13}
\end{array}\right)
$$

The general formula for the neutrino-neutrino oscillation probability is

$$
P\left(\nu_{\alpha} \rightarrow \nu_{\beta}\right)=\sum_{i j} U_{\alpha i}^{*} U_{\beta i} U_{\alpha j} U_{\beta j}^{*} e^{i\left(\overrightarrow{p_{i}}-\overrightarrow{p_{j}}\right) \vec{x}},
$$

whereas the general formula for the antineutrino-neutrino oscillation probability is

$$
P\left(\nu_{\alpha} \rightarrow \bar{\nu}_{\beta}\right)=\sum_{i j} U_{\alpha i}^{*} U_{\beta i}^{*} U_{\alpha j} U_{\beta j} \frac{m_{i} m_{j}}{p^{2}} e^{i\left(\overrightarrow{p_{i}}-\overrightarrow{p_{j}}\right) \vec{x}}
$$

As is well-known, Eq. (45) does not depend on $D_{M a j}$, while Eq. (46) does.

\subsection{Low-energy $d \leq 6$-Effective Lagrangian}

The neutrino Lagrangian for the effective theory including only $d \leq 6$ operators is given by

$$
\mathcal{L}_{\text {neutrino }}^{d \leq 6}=i \bar{\nu}_{L \alpha} \not \partial\left(\delta_{\alpha \beta}+\lambda_{\alpha \beta}\right) \nu_{L \beta}-\frac{1}{2} \overline{\nu_{L}{ }^{c}}{ }_{\alpha} m_{\alpha \beta} \nu_{L \beta}-\frac{1}{2} \bar{\nu}_{\alpha} m_{\alpha \beta}^{*} \nu_{L \beta}{ }^{c},
$$


where $m$ is defined as before, and

$$
\lambda \equiv \frac{v^{2}}{2} c^{d=6}
$$

is the contribution of the $d=6$ operator of Eq. (44) to the left-handed neutrino kinetic energy, which is flavor non-diagonal. We can rotate to the basis in which both the neutrino kinetic energy and mass matrices are diagonalized, and the neutrino field is rescaled so that the neutrino kinetic energy is normalized. In this basis, the light neutrino Majorana mass eigenstates are:

$$
\nu_{i}=V_{i \alpha}^{\mathrm{eff}} \nu_{\alpha L}+V_{i \alpha}^{\mathrm{eff} *} \nu_{\alpha L}^{c}
$$

where

$$
V^{\mathrm{eff}} \equiv V\left(\mathbb{I}-\frac{\lambda}{2}\right)
$$

depends on the unitary matrix $V$ as well as on the factor $\left(\delta_{\alpha \beta}-\frac{\lambda_{\alpha \beta}}{2}\right)$ which rescales the neutrino field in the flavor basis. $V^{\text {eff }}$ is the matrix which diagonalizes the light neutrino Majorana mass matrix ${ }^{1}$,

$$
\left(V^{\text {eff }}\right)^{*} m\left(V^{\text {eff }}\right)^{\dagger}=m_{\text {diag,real }} .
$$

Unlike $V, V^{\text {eff }}$ is not a unitary matrix because of the field rescaling. The light Majorana mass eigenstates continue to satisfy $\nu_{i}^{c}=\nu_{i}$.

The leptonic Lagrangian of the $d \leq 6$ effective theory can be rewritten in terms of the light Majorana neutrino mass eigenstates. The free neutrino Lagrangian becomes

$$
\mathcal{L}_{\text {neutrino }}^{d \leq 6}=\frac{1}{2} \overline{\nu_{i}}\left(i \overrightarrow{\not \supset}-m_{i}\right) \nu_{i}
$$

The weak currents, written in terms of the Majorana mass eigenstates, are

$$
\begin{aligned}
J_{\mu}^{-C C} & \equiv \bar{e}_{L_{\alpha}} \gamma_{\mu}\left(V^{\mathrm{eff}^{\dagger}}\right)_{\alpha i} \nu_{i}, \\
J_{\mu}^{N C} & \equiv \frac{1}{2} \bar{\nu}_{i} \gamma_{\mu}\left(V^{\mathrm{eff}}\right)_{i \alpha}\left(V^{\mathrm{eff} \dagger}\right)_{\alpha j} \nu_{j},
\end{aligned}
$$

where $V^{\text {eff }}{ }^{\dagger}$ appears in the charged current and a factor $V_{i \alpha}^{\text {eff }} V_{\alpha j}^{\text {eff }}{ }^{\dagger} \neq \delta_{i j}$ appears in the neutral current since $V^{\text {eff }}$ is not unitary.

By absorbing the $n$ phases $\omega_{\alpha}$ into the charged lepton fields as before, the physical weak currents are described by Eqs. (153) and (154) with the substitution $V^{\text {eff }}{ }^{\dagger} \rightarrow U^{\text {eff }}$, where

$$
U^{\mathrm{eff}}=\left(\mathbb{I}-\Omega \frac{\lambda}{2} \Omega^{\dagger}\right) U .
$$

The oscillation formulae are now given by Eqs. (45) and (46) with the substitution $U \rightarrow$ $U^{\text {eff }}$.

\footnotetext{
${ }^{1}$ Notice that $m$ itself is not affected by the rescaling, to this order in the $1 / M$ expansion.
} 


\subsection{Global symmetries}

The kinetic energy terms of the Standard Model theory are invariant under the chiral symmetry transformations

$$
\nu_{L} \rightarrow V_{\ell} \nu_{L}, \quad e_{L} \rightarrow V_{\ell} e_{L}, \quad e_{R} \rightarrow V_{e} e_{R}
$$

where $V_{\ell}$ and $V_{e}$ are $n \times n$ unitary matrices for $n$ generations of light fermions. The chiral symmetry group $G=U(n)_{\ell} \times U(n)_{e}$ is completely broken by the charged lepton mass matrix $m_{e}$, the light Majorana neutrino mass matrix $m$, and the light neutrino kinetic energy matrix $(\mathbb{I}+\lambda)$. The mass and kinetic energy terms would respect the chiral symmetry if the matrices $m_{e}, m$ and $\lambda$ transformed as

$$
\begin{aligned}
m_{e} & \rightarrow V_{\ell} m_{e} V_{e}^{\dagger}, \\
m & \rightarrow V_{\ell}^{*} m V_{\ell}^{\dagger}, \\
\lambda & \rightarrow V_{\ell} \lambda V_{\ell}^{\dagger},
\end{aligned}
$$

under the chiral symmetry. As shown in Ref. 9], the number of physical parameters of the low-energy effective theory including the $d=5$ and $d=6$ operators is equal to the number of parameters in the matrices $m_{e}, m$ and $\lambda$ minus the number of parameters in $V_{\ell}$ and $V_{e}$.

Any high-energy basis with $Y_{e}$ diagonal and real produces a diagonal and real charged lepton mass matrix $m_{e}$ in the spontaneously broken low-energy theory,

$$
\left(m_{e}\right)_{\text {diag,real }}=\operatorname{diag}\left(m_{e}, m_{\mu}, \cdots, m_{e_{n}}\right) .
$$

This charged lepton mass matrix is left invariant under $V_{\ell}=V_{e}$ rephasings Eq. (12) which depend on $n$ imaginary parameters, $\zeta_{\alpha}, \alpha=1, \cdots, n$.

In a general basis, the complex symmetric $n \times n$ matrix $m$ contains $n(n+1) / 2$ real and $n(n+1) / 2$ imaginary parameters defined by its matrix elements

$$
m_{\alpha \beta}=\left|m_{\alpha \beta}\right| e^{i \gamma_{\alpha \beta}}
$$

whereas the Hermitian $n \times n$ matrix $\lambda$ contains $n(n+1) / 2$ real and $n(n-1) / 2$ imaginary parameters defined by its matrix elements

$$
\lambda_{\alpha \beta}=\left|\lambda_{\alpha \beta}\right| e^{i \sigma_{\alpha \beta}}
$$

Under $\zeta_{\alpha}$ rephasings, the phases

$$
\begin{aligned}
& \gamma_{\alpha \beta} \rightarrow \gamma_{\alpha \beta}-\left(\zeta_{\alpha}+\zeta_{\beta}\right) \\
& \sigma_{\alpha \beta} \rightarrow \sigma_{\alpha \beta}+\left(\zeta_{\alpha}-\zeta_{\beta}\right)
\end{aligned}
$$

The low-energy $d \leq 6$ effective theory contains $n(n-1)$ physical, basis-independent, phases:

$$
\begin{aligned}
\bar{\gamma}_{\alpha \beta} \equiv \gamma_{\alpha \beta}-\frac{\left(\gamma_{\alpha}+\gamma_{\beta}\right)}{2}, \\
\bar{\sigma}_{\alpha \beta} \equiv \sigma_{\alpha \beta}+\frac{\left(\gamma_{\alpha}-\gamma_{\beta}\right)}{2}
\end{aligned}
$$


where $\gamma_{\alpha} \equiv \gamma_{\alpha \alpha}$ and $\gamma_{\beta} \equiv \gamma_{\beta \beta}$ are the diagonal phases of the light neutrino Majorana mass matrix $m$ in the flavor basis. The physical phases $\bar{\gamma}_{\alpha \beta}$ and $\bar{\sigma}_{\alpha \beta}$ are invariant under $\zeta_{\alpha}$ rephasings.

A physical low-energy basis expressed only in terms of the physical low-energy phases $\bar{\gamma}_{\alpha \beta}$ and $\bar{\sigma}_{\alpha \beta}$ is then defined by the choice of basis

$$
\zeta_{\alpha}=\gamma_{\alpha} / 2
$$

In the physical low-energy basis, the light neutrino mass matrix $m$ depends on the $n(n-1) / 2$ phases $\bar{\gamma}_{\alpha \beta}, \alpha \neq \beta$, and the $\lambda$ matrix depends on the $n(n-1) / 2$ phases $\bar{\sigma}_{\alpha \beta}, \alpha \neq \beta$. All in all, the basis contains a total of $n(n+2)$ real and $n(n-1)$ imaginary physical parameters in the matrices $m_{e}, m$ and $\lambda$. The number of physical low-energy parameters is equal to the number of physical parameters of the high-energy seesaw model for $n=n^{\prime}[9]$.

It is worth emphasizing that the physical low-energy basis is not identical to the standard high-energy basis, $\zeta_{\alpha}=-\Psi_{\alpha}$. The former, $\zeta_{\alpha}=\gamma_{\alpha} / 2$, is related to the latter by a relative $V_{\ell}=V_{e}$ transformation with $\zeta_{\alpha}=\Psi_{\alpha}+\gamma_{\alpha} / 2$. It is worth noting that these last phases are invariant under $V_{\ell}=V_{e}$ rephasings, as expected for a rephasing relating two physical bases: the standard high-energy and the physical low-energy bases.

\section{Connection between High- and Low-Energy Parameters}

The matrices $M$ and $Y_{\nu}$ of the high-energy seesaw model can be determined in terms of the $d=5$ and $d=6$ coefficient matrices $m$ and $\lambda$ of the broken low-energy effective theory if $n^{\prime} \leq n$. In this section, we derive explicit formulae for this connection. We first derive formulae which produce $M$ and $Y_{\nu}$ in unstandard form. The formulae for $M$ and $Y_{\nu}$ in the standard high-energy basis are related to them by a $V_{N}$ transformation.

The definitions

$$
\begin{aligned}
m & \equiv-\frac{v^{2}}{2} c^{d=5}=-\frac{v^{2}}{2}\left[Y_{\nu}^{*}\left(M^{*}\right)^{-1} Y_{\nu}^{\dagger}\right], \\
\lambda & \equiv \frac{v^{2}}{2} c^{d=6}=\frac{v^{2}}{2}\left[\left(Y_{\nu} M^{-1}\right)\left(Y_{\nu} M^{-1}\right)^{\dagger}\right],
\end{aligned}
$$

express the low-energy matrices $m$ and $\lambda$ in terms of the high-energy matrices $Y_{\nu}$ and $M$, in a basis-independent way.

For further use, we define the quantity $\chi$ through

$$
\lambda=\lambda^{\dagger}=\chi \chi^{\dagger} .
$$

One possible solution for $\chi$ is

$$
\chi \equiv-\frac{v}{\sqrt{2}} Y_{\nu} M^{-1} .
$$

Consistency with the chiral transformations given in Eqs. (10) and (57) implies that

$$
\chi \rightarrow V_{\ell} \chi V_{N}^{T} .
$$


under the chiral symmetry.

Using Eqs. (66) and (69), it is possible to solve for the high-energy matrices $Y_{\nu}$ and $M$ in terms of the low-energy matrices $m$ and $\chi$,

$$
\begin{aligned}
Y_{\nu} & =\frac{\sqrt{2}}{v} m^{\dagger}\left(\chi^{-1}\right)^{T}, \\
M & =-\left(\chi^{-1}\right) m^{\dagger}\left(\chi^{-1}\right)^{T} .
\end{aligned}
$$

This model-independent determination of the seesaw parameters $Y_{\nu}$ and $M$ requires the knowledge of both $m$ and $\chi$, and thus it is only possible when both the $d=5$ and $d=6$ coefficients are measured at low energies. A priori, one can deduce all of the physical highenergy parameters from hypothetical measurements of the low-energy parameters. The caveat refers to the practical difficulty of measuring the $d=6$ coefficients, for natural values of the seesaw scale. As we discuss in detail momentarily, the matrices $Y_{\nu}$ and $M$ given by Eqs. (71) and (72) do not correspond to the standard high-energy basis.

The lepto-asymmetry parameter which is relevant for leptogenesis depends on the highenergy matrices $M$ and $Y_{\nu}$. In a general basis, it depends on both the phases of the heavy Majorana neutrino mass matrix $M$, Eq. (72), and the phases of the matrix elements of

$$
Y_{\nu}^{\dagger} Y_{\nu}=\frac{2}{v^{2}}\left(\chi^{-1}\right)^{*} m m^{\dagger}\left(\chi^{-1}\right)^{T}
$$

Notice that this expression is invariant under $V_{\ell}$ transformations, while it depends on the high-energy basis chosen for the heavy Majorana neutrinos, transforming under $V_{N}$ transformations as

$$
Y_{\nu}^{\dagger} Y_{\nu} \rightarrow V_{N} Y_{\nu}^{\dagger} Y_{\nu} V_{N}^{\dagger}
$$

There is an important subtlety to the above derivation of the high-energy matrices. The explicit expressions Eqs. (71) and (72) for $M$ and $Y_{\nu}$ depend on the low-energy basis chosen for $m$ and $\lambda$. For instance, when the matrices $m$ and $\lambda$ are written in the physical low-energy basis, the heavy neutrino mass matrix $M$ obtained using Eq. (72) is neither diagonal nor real, as it is, by definition, in the standard high-energy basis. A further $V_{N}$ transformation is required to obtain the standard high-energy basis with $M$ diagonal and real.

The point is that the definition of $\chi$ given in Eq. (69) is not unique. It is clear that multiplying the right-hand side of Eq. (69) by any unitary matrix,

$$
\chi \rightarrow \chi V_{N}^{T},
$$

results in an equally valid solution for $\chi$, since it produces the same $\lambda$ matrix as Eq. (69). The unitary matrix is denoted by $V_{N}$ to maintain consistency with the chiral transformations given in Eqs. (10) and (57). Different $V_{N}$ transformations correspond to different choices of the high energy basis, as $Y_{\nu} \rightarrow Y_{\nu} V_{N}^{\dagger}$ and $M \rightarrow V_{N}{ }^{*} M V_{N}^{\dagger}$. The specific $V_{N}$ which makes $M$ diagonal and real yields the standard high-energy basis.

Let us consider more explicitly the low-energy basis. The $m$ matrix is diagonalized by the unitary matrix $V$ as defined in Eq. (35). Let us define the unitary matrix $W$ which diagonalizes the $\lambda$ matrix by

$$
\lambda_{\text {diag, real }} \equiv W^{\dagger} \lambda W
$$


From Eq. (68), the general solution for $\chi$ is given by

$$
\chi=W \sqrt{\lambda_{\text {diag, real }}} V_{N}^{T} .
$$

$Y_{\nu}$ and $M$ and the matrix $Y_{\nu}^{\dagger} Y_{\nu}$ relevant for leptogenesis now can be expressed in all generality $\mathrm{as}^{2}$

$$
\begin{aligned}
Y_{\nu} & =\frac{\sqrt{2}}{v} V^{\dagger} m_{\text {diag, real }} L, \\
M & =-L^{T} m_{\text {diag,real }} L, \\
Y_{\nu}^{\dagger} Y_{\nu} & =\frac{2}{v^{2}} L^{\dagger} m_{\text {diag,real }}^{2} L,
\end{aligned}
$$

where the matrix $L$ is given by

$$
L \equiv\left(\chi^{-1} V^{\dagger}\right)^{T}=V^{*} W^{*}\left(\sqrt{\lambda_{\text {diag, real }}}\right)^{-1} V_{N}^{\dagger} .
$$

The standard high-energy basis is obtained if $V_{N}$ is chosen to be the unitary matrix which diagonalizes $M$ in Eq. (179). Thus, $V_{N}$ is determined a priori from low-energy measurements,

$$
M_{\text {diag, real }} \equiv-V_{N}^{*}\left(\left(\sqrt{\lambda_{\text {diag, real }}}\right)^{-1} W^{\dagger} V^{\dagger} m_{\text {diag, real }} V^{*} W^{*}\left(\sqrt{\lambda_{\text {diag, real }}}\right)^{-1}\right) V_{N}^{\dagger} .
$$

It is easy to show a number of interesting results using the solutions Eqs. (71) and (72) for $Y_{\nu}$ and $M$. For example, if there is no $C P$-violation at low energies, then there is a low-energy basis in which $m$ and $\lambda$ are both real. From the definitions in Eqs. (35) and (76), it follows that $V$ and $W$ are real, as are $V_{N}$ in Eq. (82) and therefore $\chi$ in Eq. (777). Thus, it follows from Eqs. (71) and (72) that $Y_{\nu}$ and $M$ are real, and there is no leptogenesis at high energies.

Eqs. (82) simplifies considerably for a $\lambda$ matrix with equal eigenvalues, $\lambda_{i}=|\lambda|$. The heavy neutrino masses then are given by

$$
M_{\text {diag,real }}=-\frac{1}{|\lambda|} V_{N}^{*} V^{\dagger} m_{\text {diag,real }} V^{*} V_{N}^{\dagger}=-\frac{m_{\text {diag,real }}}{|\lambda|},
$$

where $W=1, V_{N}=V^{*}$, and Eq. (81) reduces to $L=\mathbb{I} / \sqrt{|\lambda|}$. In other words, the heavy neutrino masses are proportional to the light neutrino masses when $\lambda$ has degenerate eigenvalues. Eq. (83) further implies that the heavy Majorana neutrino masses become degenerate, $\left|M_{i}\right|=|M|$, in the limit when both the light neutrinos masses and the $\lambda$-eigenvalues become degenerate, $m_{i}=|m|$ and $\lambda_{i}=|\lambda|$ :

$$
|M|=\frac{|m|}{|\lambda|} .
$$

Let us now turn to the analysis of the lepto-asymmetries in the basis in which $M$ is diagonal and real. From Eq. (80), it follows that leptogenesis vanishes in the following two limits:

\footnotetext{
${ }^{2}$ The results in this section can be easily rewritten in terms of the usual PMNS matrix, with the help of Eq. (40). This is done for the two generation case in section 5 .
} 
- Degenerate eigenvalues $\lambda_{i}=|\lambda|$ of the $d=6$ matrix $\lambda$. In this limit, the matrix $L=\mathbb{I} / \sqrt{|\lambda|}$, and $Y_{\nu}^{\dagger} Y_{\nu}$ in Eq. (80) is real and diagonal,

$$
Y_{\nu}^{\dagger} Y_{\nu}=\frac{2}{v^{2}} \frac{m_{\text {diag,real }}^{2}}{|\lambda|}
$$

- Degenerate light neutrino masses $m_{i}=|m|$, when the heavy neutrinos are strongly hierarchical. For $M_{1} \ll M_{2}, M_{3}, \cdots, M_{n^{\prime}}$, the lepto-asymmetry $\epsilon_{1}$ given in Eq. (23) is approximated by [17]

$$
\begin{aligned}
\epsilon_{1} & \simeq-\frac{3}{8 \pi} \frac{\left|M_{1}\right|}{\left[Y_{\nu}^{\dagger} Y_{\nu}\right]_{11}} \sum_{j \neq 1}^{n^{\prime}} \frac{\operatorname{Im}\left[\left(Y_{\nu}^{\dagger} Y_{\nu}\right)_{1 j}\right]^{2}}{\left|M_{j}\right|} \\
& =-\frac{3}{8 \pi} \frac{1}{\left[Y_{\nu}^{\dagger} Y_{\nu}\right]_{11}} \sum_{j \neq 1}^{n^{\prime}} \operatorname{Im}\left\{\frac{M_{1}}{M_{j}}\left[\left(Y_{\nu}^{\dagger} Y_{\nu}\right)_{1 j}\right]^{2}\right\} \\
& =\frac{3}{4 \pi} \frac{1}{v^{2}} \frac{1}{\left[Y_{\nu}^{\dagger} Y_{\nu}\right]_{11}} \operatorname{Im}\left\{M_{1}\left[Y_{\nu}^{\dagger} m^{\dagger} Y_{\nu}^{*}\right]_{11}\right\}
\end{aligned}
$$

or, in terms of $L$ using Eqs. (78) and (79),

$$
\epsilon_{1} \simeq-\frac{3}{4 \pi} \frac{1}{v^{2}} \frac{\operatorname{Im}\left\{\left[L^{T} m_{\text {diag,real }} L\right]_{11}\left[L^{\dagger} m_{\text {diag,real }}^{3} L^{*}\right]_{11}\right\}}{\left[L^{\dagger} m_{\text {diag,real }}^{2} L\right]_{11}} .
$$

In the limit when all the light neutrinos have the same mass, i.e. $m_{\text {diag, real }}=|m| \mathbb{I}$, the $C P$-odd lepto-asymmetry vanishes:

$$
\epsilon_{1} \simeq-\frac{3}{4 \pi} \frac{|m|^{2}}{v^{2}} \frac{1}{\left[L^{\dagger} L\right]_{11}} \operatorname{Im}\left\{\left[L^{T} L\right]_{11}\left[L^{T} L\right]_{11}^{\dagger}\right\}=0
$$

since the diagonal matrix elements of a Hermitian matrix are real.

The lepton asymmetry in Eq. (87) can be rewritten in terms of the squared mass differences of the light neutrino masses [15, 17] to emphasize that it vanishes in the limit of degenerate light neutrino masses,

$$
\begin{aligned}
\epsilon_{1} & =-\frac{3}{4 \pi} \frac{1}{v^{2}} \frac{\left[L^{T} m_{\text {diag,real }} L\right]_{11}}{\left[L^{\dagger} m_{\text {diag,real }}^{2} L\right]_{11}} \sum_{j} m_{j}^{3} \operatorname{Im}\left\{\left[L^{*}\right]_{j 1}^{2}\right\} \\
& =-\frac{3}{4 \pi} \frac{1}{v^{2}} \frac{\left[L^{T} m_{\text {diag,real }} L\right]_{11}}{\left[L^{\dagger} m_{\text {diag,real }}^{2} L\right]_{11}} \sum_{j} m_{j} \Delta m_{j 1}^{2} \operatorname{Im}\left\{\left[L^{*}\right]_{j 1}^{2}\right\}
\end{aligned}
$$

where we have used

$$
m_{1} \operatorname{Im}\left\{M_{1}\right\}=m_{1} \sum_{j} m_{j} \operatorname{Im}\left\{\left[L^{*}\right]_{j 1}^{2}\right\}=0 .
$$




\subsection{Connection with the literature}

Many different parameterizations for the Yukawa couplings of the heavy Majorana neutrinos can be found in the literature. Our treatment is an improvement over earlier ones in that no matrix with undefined matrix elements is used. Instead, all high-energy parameters are derived in terms of matrix elements of the $d=5$ and $d=6$ operator coefficients. These matrix elements can be determined a priori from low-energy measurements; indeed, we have related all $d=5$ and $d=6$ matrix elements to $C P$-odd and $C P$-even observables.

A very popular parameterization is that in which $Y_{\nu}$ is expressed in terms of an orthogonal matrix $R[18$,

$$
Y_{\nu}=V^{\dagger} \sqrt{m_{\text {diag, real }}} R^{\dagger} \sqrt{\left|M_{\text {diag, real }}\right|}
$$

The combination relevant for leptogenesis then is written as

$$
Y_{\nu}^{\dagger} Y_{\nu}=\sqrt{\left|M_{\text {diag, real }}\right|} R m_{\text {diag, real }} R^{\dagger} \sqrt{\left|M_{\text {diag, real }}\right|} .
$$

$R$ is left arbitrary in the usual treatment which only includes the effects of the $d=5$ operator. Within this treatment, all the $C P$-violating phases are embedded in $R$, and are, therefore, unknown. The $d \leq 6$-effective theory instead allows $R$ to be determined in terms of the $d=5$ and $d=6$ coefficients. From a comparison of Eqs. (78) and (92), we obtain

$$
R=\frac{\sqrt{2}}{v}\left(\sqrt{\left|M_{\text {diag, real }}\right|}\right)^{-1} L^{\dagger} \sqrt{m_{\text {diag, real }}} .
$$

Notice that $R$ is real whenever $L$ is real.

In a different approach [19], called the bi-unitary parameterization, matrices $U_{L}$ and $U_{R}$ are defined such that

$$
Y_{\nu}=U_{L}^{\dagger}\left(Y_{\nu}\right)_{\text {diag,real }} U_{R}
$$

Comparison with the chiral transformations in Eq. (10) shows that $U_{L}$ corresponds to a particular choice of $V_{\ell}$, while $U_{R}$ belongs to the class of $V_{N}$ transformations. Notice, though, that $U_{R}$ does not correspond to the $V_{N}$ transformation which diagonalizes $M$ but to the diagonalization of $Y_{\nu}^{\dagger} Y_{\nu}$, with $M$, in general, remaining complex and non-diagonal. From our Eqs. (80) and (81), the dependence of $U_{R}$ and $U_{L}$ on the low-energy mixing parameters can be extracted.

A different series of works [20] takes advantage of the properties of triangular matrices, defining

$$
Y_{\nu}=\mathbf{U} Y_{\Delta}
$$

where $\mathbf{U}$ is unitary and $Y_{\Delta}$ triangular. $\mathbf{U}$ now can be identified with yet another particular $V_{\ell}$ transformation.

Finally, let us consider the matrix $Y_{\nu} Y_{\nu}^{\dagger}$. In supersymmetric versions of the seesaw mechanism, renormalization effects due to the neutrino Yukawa interaction induce flavor-mixing terms in the slepton mass matrices [21, resulting in lepton flavor-violating decays [18, 22]. 
The relevant quantities are the off-diagonal matrix elements of $Y_{\nu} Y_{\nu}^{\dagger}$. In the minimal, nonsupersymmetric, seesaw model, this combination can be written in terms of the low-energy observables as

$$
\begin{aligned}
Y_{\nu} Y_{\nu}^{\dagger} & =\frac{2}{v^{2}} m^{\dagger}\left(\chi^{-1}\right)^{T}\left(\chi^{-1}\right)^{*} m \\
& =\frac{2}{v^{2}} V^{\dagger} m_{\text {diag,real }} L L^{\dagger} m_{\text {diag,real }} V .
\end{aligned}
$$

In the limit of degenerate eigenvalues of the $d=6$ coefficient matrix, the only mixing parameters which remain in Eq. (97) are the usual neutrino mixing parameters stemming from the $d=5$ operator,

$$
Y_{\nu} Y_{\nu}^{\dagger}=\frac{2}{v^{2}} \frac{1}{|\lambda|} V^{\dagger} m_{\text {diag,real }}^{2} V
$$

In fact, $Y_{\nu} Y_{\nu}^{\dagger}$ is a quantity which depends on the low-energy basis chosen, as can be seen from its chiral transformation properties under $V_{\ell}$ rephasings,

$$
Y_{\nu} Y_{\nu}^{\dagger} \rightarrow V_{\ell} Y_{\nu} Y_{\nu}^{\dagger} V_{\ell}^{\dagger}
$$

whereas it is invariant under $V_{N}$ chiral transformations. This behavior is opposite to that of the combination relevant for leptogenesis $Y_{\nu}^{\dagger} Y_{\nu}$, see Eq. (744).

\section{$5 \quad$ Two generations}

In this section, we explicitly relate the parameters of the high-energy seesaw model to the lowenergy parameters of the effective theory in the case of two generations of heavy neutrinos and Standard Model leptons. In this case, the leptonic sectors of the high-energy seesaw Lagrangian and the low-energy $d \leq 6$-effective Lagrangian each contain 8 real and 2 imaginary parameters.

\subsection{High-energy Seesaw Lagrangian}

The seesaw model with two lepton generations is defined by $2 \times 2$ matrices $Y_{e}, M$ and $Y_{\nu}$. In the standard high-energy basis, $Y_{e}$ and $M$ are the diagonal and real matrices

$$
\begin{gathered}
\left(Y_{e}\right)_{\text {diag, real }}=\left(\begin{array}{cc}
y_{e} & 0 \\
0 & y_{\mu}
\end{array}\right) \equiv \frac{\sqrt{2}}{v}\left(\begin{array}{cc}
m_{e} & 0 \\
0 & m_{\mu}
\end{array}\right), \\
M_{\text {diag, real }}=\left(\begin{array}{cc}
\left|M_{1}\right| & 0 \\
0 & \left|M_{2}\right|
\end{array}\right)
\end{gathered}
$$

whereas the matrix $Y_{\nu}$ is given by

$$
Y_{\nu}=\left(\begin{array}{cc}
u_{e 1} e^{-i \Phi_{e 2}} & u_{e 2} e^{i \Phi_{e 2}} \\
u_{\mu 1} e^{i \Phi_{\mu}} & u_{\mu 2} e^{-i \Phi_{\mu}}
\end{array}\right) \equiv\left(\begin{array}{cc}
u_{1} e^{-i \Phi_{e}} & u_{2} e^{i \Phi_{e}} \\
v_{1} e^{i \Phi_{\mu}} & v_{2} e^{-i \Phi_{\mu}}
\end{array}\right)
$$


For simplicity, the notation defined by the right-hand side of this equation will be used hereafter instead of the general notation displayed on the left-hand side of the equation.

The 2 physical phases of the high-energy theory are $\Phi_{e}$ and $\Phi_{\mu}$, whereas the 8 real parameters are $y_{e}, y_{\mu}, M_{1}, M_{2}, u_{1}, v_{1}, u_{2}$ and $v_{2}$. The charged lepton Yukawa parameters $y_{e}$ and $y_{\mu}$ are not free parameters, but are known in terms of the charged lepton masses and the Higgs vacuum expectation value.

Leptogenesis at high energy depends on the $C P$ asymmetries produced in heavy Majorana neutrino decay. The $C P$ asymmetry produced by decay of $N_{1}$ is

$$
\epsilon_{1}=\frac{1}{\pi\left(Y_{\nu}^{\dagger} Y_{\nu}\right)_{11}} \operatorname{Im}\left[\left(Y_{\nu}^{\dagger} Y_{\nu}\right)_{12}\right]^{2} f\left(\frac{\left|M_{2}\right|}{\left|M_{1}\right|}\right)
$$

which is equal to

$$
\epsilon_{1}=\frac{1}{\pi\left(u_{1}^{2}+v_{1}^{2}\right)} \operatorname{Im}\left[\left(u_{1} u_{2} e^{2 i \Phi_{e}}+v_{1} v_{2} e^{-2 i \Phi_{\mu}}\right)^{2}\right] f\left(\frac{\left|M_{2}\right|}{\left|M_{1}\right|}\right),
$$

in the standard high-energy basis.

\section{$5.2 d=5$ and $d=6$ coefficients}

The $d=5$ and $d=6$ operator coefficients of the low-energy effective Lagrangian, $c^{d=5}$ and $c^{d=6}$, are defined in terms of the matrices $M$ and $Y_{\nu}$ of the high-energy seesaw Lagrangian by Eqs. (3) and (51) [9]. In the standard high-energy basis, they are

$$
c^{d=5}=\left(\begin{array}{cc}
\frac{u_{1}^{2}}{M_{1}} e^{i 2 \Phi_{e}}+\frac{u_{2}^{2}}{M_{2}} e^{-i 2 \Phi_{e}} & \frac{u_{1} v_{1}}{M_{1}} e^{i\left(\Phi_{e}-\Phi_{\mu}\right)}+\frac{u_{2} v_{2}}{M_{2}} e^{-i\left(\Phi_{e}-\Phi_{\mu}\right)} \\
\frac{u_{1} v_{1}}{M_{1}} e^{i\left(\Phi_{e}-\Phi_{\mu}\right)}+\frac{u_{2} v_{2}}{M_{2}} e^{-i\left(\Phi_{e}-\Phi_{\mu}\right)} & \frac{v_{1}^{2}}{M_{1}} e^{-i 2 \Phi_{\mu}}+\frac{v_{2}^{2}}{M_{2}} e^{i 2 \Phi_{\mu}}
\end{array}\right)
$$

and

$$
c^{d=6}=\left(\begin{array}{cc}
\frac{u_{1}^{2}}{M_{1}^{2}}+\frac{u_{2}^{2}}{M_{2}^{2}} & \frac{u_{1} v_{1}}{M_{1}^{2}} e^{-i\left(\Phi_{e}+\Phi_{\mu}\right)}+\frac{u_{2} v_{2}}{M_{2}^{2}} e^{i\left(\Phi_{e}+\Phi_{\mu}\right)} \\
\frac{u_{1} v_{1}}{M_{1}^{2}} e^{i\left(\Phi_{e}+\Phi_{\mu}\right)}+\frac{u_{2} v_{2}}{M_{2}^{2}} e^{-i\left(\Phi_{e}+\Phi_{\mu}\right)} & \frac{v_{1}^{2}}{M_{1}^{2}}+\frac{v_{2}^{2}}{M_{2}^{2}}
\end{array}\right) .
$$

The low-energy real and imaginary parameters of the $d=5$ and $d=6$ coefficient matrices are defined by

$$
\begin{aligned}
c^{d=5} & \equiv-\left(\begin{array}{cc}
\left|c_{e e}^{d=5}\right| e^{i \gamma_{e}} & \left|c_{e \mu}^{d=5}\right| e^{i \gamma_{e \mu}} \\
\left|c_{e \mu}^{d=5}\right| e^{i \gamma_{e \mu}} & \left|c_{\mu \mu}^{d=5}\right| e^{i \gamma_{\mu}}
\end{array}\right), \\
c^{d=6} & \equiv\left(\begin{array}{cc}
\left|c_{e e}^{d=6}\right| & \left|c_{e \mu}^{d=6}\right| e^{i \sigma_{e \mu}} \\
\left|c_{e \mu}^{d=6}\right| e^{-i \sigma_{e \mu}} & \left|c_{\mu \mu}^{d=6}\right|
\end{array}\right) .
\end{aligned}
$$

(An explicit minus sign has been introduced into the definition of the $d=5$ operator coefficient parameters to simplify later formulae for the light neutrino Majorana mass matrix.) 
The low-energy phases $\gamma_{e}, \gamma_{\mu}, \gamma_{e \mu}$ of the $d=5$ coefficient and $\sigma_{e \mu}$ of the $d=6$ coefficient can be expressed in terms of the parameters of the high-energy standard basis by

$$
\begin{aligned}
\gamma_{e}=\arctan \left(\left(\frac{\frac{u_{1}^{2}}{M_{1}}-\frac{u_{2}^{2}}{M_{2}}}{\frac{u_{1}^{2}}{M_{1}}+\frac{u_{2}^{2}}{M_{2}}}\right) \tan 2 \Phi_{e}\right)+\pi, \\
\gamma_{\mu}=\arctan \left(\left(\frac{\frac{v_{2}^{2}}{M_{2}}-\frac{v_{1}^{2}}{M_{1}}}{\frac{v_{2}^{2}}{M_{2}}+\frac{v_{1}^{2}}{M_{1}}}\right) \tan 2 \Phi_{\mu}\right)+\pi, \\
\gamma_{e \mu}=\arctan \left(\left(\frac{\frac{u_{2} v_{2}}{M_{2}}-\frac{u_{1} v_{1}}{M_{1}}}{\frac{u_{2} v_{2}}{M_{2}}+\frac{u_{1} v_{1}}{M_{1}}}\right) \tan \left(\Phi_{e}-\Phi_{\mu}\right)\right)+\pi, \\
\sigma_{e \mu}=\arctan \left(\left(\frac{\frac{u_{2} v_{2}}{M_{2}^{2}}-\frac{u_{1} v_{1}}{M_{1}^{2}}}{\frac{u_{2} v_{2}}{M_{2}^{2}}+\frac{u_{1} v_{1}}{M_{1}^{2}}}\right) \tan \left(\Phi_{e}+\Phi_{\mu}\right)\right) .
\end{aligned}
$$

There are only 2 independent low-energy phases which are invariant under $\zeta_{\alpha}$ rephasings at low energies:

$$
\bar{\gamma} \equiv \gamma_{e \mu}-\frac{\gamma_{e}+\gamma_{\mu}}{2}
$$

and

$$
\bar{\sigma} \equiv \sigma_{e \mu}+\frac{\gamma_{e}-\gamma_{\mu}}{2}
$$

The phases $\bar{\gamma}$ and $\bar{\sigma}$ are the 2 physical phases of the low-energy $d \leq 6$-effective Lagrangian which cannot be eliminated by a change of basis. In the following, when considering the lowenergy effective theory, we will work in the basis where all the unphysical phases are removed by performing a rephasing

$$
V_{e}=V_{\ell}=\left(\begin{array}{cc}
e^{i \gamma_{e} / 2} & 0 \\
0 & e^{i \gamma_{\mu} / 2}
\end{array}\right)
$$

relative to the standard high-energy basis, so that only the physical phases $\bar{\gamma}$ and $\bar{\sigma}$ appear. In this physical low-energy basis, the $d=5$ and $d=6$ coefficients are given by

$$
\begin{aligned}
c^{d=5} & \equiv-\left(\begin{array}{cc}
\left|c_{e e}^{d=5}\right| & \left|c_{e \mu}^{d=5}\right| e^{i \bar{\gamma}} \\
\left|c_{e \mu}^{d=5}\right| e^{i \bar{\gamma}} & \left|c_{\mu \mu}^{d=5}\right|
\end{array}\right), \\
c^{d=6} & \equiv\left(\begin{array}{cc}
\left|c_{e e}^{d=6}\right| & \left|c_{e \mu}^{d=6}\right| e^{i \bar{\sigma}} \\
\left|c_{e \mu}^{d=6}\right| e^{-i \bar{\sigma}} & \left|c_{\mu \mu}^{d=6}\right|
\end{array}\right) .
\end{aligned}
$$

We will see that this basis is particularly useful for relating high- and low-energy observables. In particular, explicit formulae that express the $2 C P$-odd phases a priori measurable in the experiments in terms of $\bar{\gamma}$ and $\bar{\sigma}$ are given later. Through Eqs. (107)-(109), the high-energy phases $\Phi_{e}$ and $\Phi_{\mu}$ can be directly related to the observable low-energy $C P$-odd phases. 


\subsection{Low-energy Effective Lagrangian}

The parameters of the low-energy effective theory including the $d=5$ and $d=6$ operators are given by the two charged lepton masses $m_{e}$ and $m_{\mu}$, the two light neutrino Majorana masses $m_{1}$ and $m_{2}$, the mixing angle $\theta$, the phase of the lepton mixing matrix, the 3 magnitudes $\lambda_{e e}$, $\lambda_{e \mu}$ and $\lambda_{\mu \mu}$, and the phase of the $\lambda$ matrix. These parameters are defined in this subsection.

The light Majorana neutrino masses and lepton mixing matrix are derived first in the effective theory including only the $d=5$ operator, and then in the effective theory including both the $d=5$ and $d=6$ operators.

\subsubsection{Low-energy $d \leq 5$-Effective Lagrangian}

Consider the neutrino Lagrangian in Eq. (33). In the physical low-energy basis, the complex symmetric $2 \times 2$ mass matrix of the light neutrinos depends on 3 real and 1 imaginary parameters:

$$
m \equiv-\frac{v^{2}}{2} c^{d=5}=\left(\begin{array}{cc}
m_{e e} & m_{e \mu} e^{i \bar{\gamma}} \\
m_{e \mu} e^{i \bar{\gamma}} & m_{\mu \mu}
\end{array}\right)
$$

where the $m_{\alpha \beta}$ are real and positive. The light neutrino Majorana mass matrix is diagonalized by the transformation

$$
m \rightarrow V^{*} m V^{\dagger}=m_{\text {diag,real }}=\left(\begin{array}{cc}
m_{1} & 0 \\
0 & m_{2}
\end{array}\right)
$$

where the $2 \times 2$ unitary matrix $V$ contains 1 real and 3 imaginary parameters which are parameterized by a real mixing angle and 3 phases

$$
V^{\dagger}=\left(\begin{array}{cc}
e^{-i \hat{\theta}_{e} / 2} & 0 \\
0 & e^{-i \hat{\theta}_{\mu} / 2}
\end{array}\right)\left(\begin{array}{cc}
\cos \theta & \sin \theta e^{-i \phi / 2} \\
-\sin \theta e^{i \phi / 2} & \cos \theta
\end{array}\right) .
$$

The mixing angle $\theta$ is taken to be in the interval $[0, \pi]$.

A discussion of the diagonalization of the low-energy $2 \times 2$ Majorana mixing matrix can be found in Ref. [23]. There, the unitary matrix is given by ${ }^{3}$

$$
V^{\dagger}=\left(\begin{array}{cc}
\cos \theta & \sin \theta e^{-i \rho} \\
-\sin \theta e^{i \rho} & \cos \theta
\end{array}\right)\left(\begin{array}{cc}
e^{-i \hat{\theta}_{e} / 2} & 0 \\
0 & e^{-i \hat{\theta}_{\mu} / 2}
\end{array}\right),
$$

with

$$
\rho \equiv \frac{\phi+\hat{\theta}_{e}-\hat{\theta}_{\mu}}{2}
$$

The 1 real and 3 imaginary parameters in $V$ are determined by the diagonalization of the light neutrino Majorana mass matrix, Eq. (114). Under a $V_{\ell}=V_{e}$ rephasing, the three phases $\phi($ or $\rho), \hat{\theta}_{e}$ and $\hat{\theta}_{\mu}$ are invariant.

\footnotetext{
${ }^{3}$ The phases $\bar{\gamma}$ and $\rho$, and the mixing angle $\theta$ defined in this work correspond to the phases $\beta$ and $-\alpha$, and the angle $-\theta$ in Ref. 23 .
} 
The $\hat{\theta}_{\alpha}$ phases are not directly measurable when considering only the $d \leq 5$ effective Lagrangian, since they can be removed from the Lagrangian by performing a rephasing on the charged lepton fields, Eq. (40),

$$
\Omega\left(\begin{array}{c}
e \\
\mu
\end{array}\right)_{L, R}=\left(\begin{array}{cc}
e^{i \omega_{e}} & 0 \\
0 & e^{i \omega_{\mu}}
\end{array}\right)\left(\begin{array}{c}
e \\
\mu
\end{array}\right)_{L, R} \rightarrow\left(\begin{array}{c}
e \\
\mu
\end{array}\right)_{L, R},
$$

which leaves all terms in the leptonic Lagrangian invariant, with the exception of the weak charged current. The particular choice

$$
\omega_{e}=\left(\hat{\theta}_{e}+\phi\right) / 2, \quad \omega_{\mu}=\hat{\theta}_{\mu} / 2
$$

yields the weak charged current

$$
J_{\mu}^{-C C}=\bar{e}_{L \alpha} \gamma_{\mu} U_{\alpha i} \nu_{i}
$$

in terms of the usual leptonic mixing matrix

$$
U=\left(\begin{array}{cc}
\cos \theta & \sin \theta \\
-\sin \theta & \cos \theta
\end{array}\right)\left(\begin{array}{cc}
e^{i \phi / 2} & 0 \\
0 & 1
\end{array}\right)
$$

It will not be possible to eliminate the two $\hat{\theta}_{\alpha}$ phases when the $d=6$ operator is included in the low-energy effective Lagrangian. Indeed, we show below that the $\hat{\theta}_{\alpha}$ phases are essential for relating the physical low-energy phases $\bar{\gamma}$ and $\bar{\sigma}$ with the $C P$-odd phases measurable in experiments. We will see in the next subsections that the $\hat{\theta}_{\alpha}$ phases are functions of the mass eigenvalues $m_{1}$ and $m_{2}$, the mixing angle $\theta$ and the phase $\phi$ (or $\rho$ ), which is as expected since the original matrix $m$ only contained one physical phase.

Note that it is not possible to remove the phase $\phi$ (or, equivalently, $\rho$ in the alternative parameterization of $V$ ) from the effective theory by rephasings on the $\nu_{i}$, since these rephasings destroy the relations $\nu_{i}^{c}=\nu_{i}$, introducing additional phase factors into these relations, i.e. $\nu_{1}^{c}=e^{-i \phi} \nu_{1}$. These phase factors contribute to physical observables, yielding results equivalent to keeping $\phi$ in the lepton mixing matrix. Throughout we stick to the convention that $\nu_{i}^{c}=\nu_{i}$, so that rephasings of the light Majorana neutrinos are forbidden.

\subsubsection{Mass vs. Flavor Eigenstate Basis at Low Energy}

Here, we give a brief summary of the relationship of the mass matrix parameters in the flavor eigenstate and mass eigenstate bases. From Eq. (35),

$$
\begin{aligned}
m_{e e} & =\left[m_{1} \cos ^{2} \theta+m_{2} \sin ^{2} \theta e^{i \phi}\right] e^{i \hat{\theta}_{e}}, \\
m_{\mu \mu} & =\left[m_{1} \sin ^{2} \theta e^{-i \phi}+m_{2} \cos ^{2} \theta\right] e^{i \hat{\theta}_{\mu}}, \\
m_{e \mu} e^{i \bar{\gamma}} & =\left(m_{2} e^{i \phi / 2}-m_{1} e^{-i \phi / 2}\right) \cos \theta \sin \theta e^{i \frac{\hat{\theta}_{e}+\hat{\theta}_{\mu}}{2}} .
\end{aligned}
$$


From the vanishing of the imaginary parts of the r.h.s. of Eqs. (122) and (123), we deduce the following relations between the phases $\hat{\theta}_{\alpha}$ and the physical observables

$$
\begin{aligned}
\cot \hat{\theta}_{e} & =-\cot \phi-\frac{m_{1}}{m_{2}} \frac{\cot ^{2} \theta}{\sin \phi}, \\
\cot \hat{\theta}_{\mu} & =\cot \phi+\frac{m_{2}}{m_{1}} \frac{\cot ^{2} \theta}{\sin \phi} .
\end{aligned}
$$

Thus, the phases $\hat{\theta}_{\alpha}$ are functions of the four independent parameters $m_{1}, m_{2}$, the mixing angle $\theta$ and the phase $\phi$. Notice that the phase $\rho$ of the parameterization in Eqs. (116) and (117), is also a function of these parameters, and in consequence, it can be determined only after all of the parameters have been measured in low-energy experiments.

The real and imaginary parts of Eq. (124) yield

$$
\begin{aligned}
& m_{e \mu} \cos \bar{\gamma}=\frac{1}{2} \sin 2 \theta\left[\left(m_{2}-m_{1}\right) \cos \frac{\phi}{2} \cos \frac{\hat{\theta}_{e}+\hat{\theta}_{\mu}}{2}-\left(m_{1}+m_{2}\right) \sin \frac{\phi}{2} \sin \frac{\hat{\theta}_{e}+\hat{\theta}_{\mu}}{2}\right], \\
& m_{e \mu} \sin \bar{\gamma}=\frac{1}{2} \sin 2 \theta\left[\left(m_{2}-m_{1}\right) \cos \frac{\phi}{2} \sin \frac{\hat{\theta}_{e}+\hat{\theta}_{\mu}}{2}+\left(m_{1}+m_{2}\right) \sin \frac{\phi}{2} \cos \frac{\hat{\theta}_{e}+\hat{\theta}_{\mu}}{2}\right],
\end{aligned}
$$

from which we deduce the expression that links the phase $\bar{\gamma}$ with the physical observables:

$$
\tan \bar{\gamma}=\frac{\left(m_{2}-m_{1}\right) \tan \frac{\hat{\theta}_{e}+\hat{\theta}_{\mu}}{2}+\left(m_{1}+m_{2}\right) \tan \frac{\phi}{2}}{\left(m_{2}-m_{1}\right)-\left(m_{1}+m_{2}\right) \tan \frac{\hat{\theta}_{e}+\hat{\theta}_{\mu}}{2} \tan \frac{\phi}{2}} .
$$

Additional details of the connection between light neutrino mass and flavor eigenstates, based on the analysis of Ref. [23, are given in Appendix 2.

\subsubsection{Oscillation probabilities for $d \leq 5$-Effective Theory}

For two generations of leptons, the neutrino-neutrino oscillation probability is given explicitly by

$$
P\left(\nu_{e} \rightarrow \nu_{\mu}\right)=\sin ^{2} 2 \theta \sin ^{2}\left(\frac{\Delta m^{2} L}{4 E}\right)=\left(1-P\left(\nu_{e} \rightarrow \nu_{e}\right)\right)
$$

and the neutrino-antineutrino oscillation probabilities are [24]:

$$
\begin{aligned}
P\left(\nu_{e} \rightarrow \bar{\nu}_{e}\right) & =\frac{m_{1}^{2}}{E^{2}} \cos ^{2} \theta+\frac{m_{2}^{2}}{E^{2}} \sin ^{2} \theta-P\left(\nu_{e} \rightarrow \bar{\nu}_{\mu}\right), \\
P\left(\bar{\nu}_{e} \rightarrow \nu_{e}\right) & =\frac{m_{1}^{2}}{E^{2}} \cos ^{2} \theta+\frac{m_{2}^{2}}{E^{2}} \sin ^{2} \theta-P\left(\bar{\nu}_{e} \rightarrow \nu_{\mu}\right), \\
P\left(\nu_{\mu} \rightarrow \bar{\nu}_{\mu}\right) & =\frac{m_{1}^{2}}{E^{2}} \sin ^{2} \theta+\frac{m_{2}^{2}}{E^{2}} \cos ^{2} \theta-P\left(\nu_{\mu} \rightarrow \bar{\nu}_{e}\right), \\
P\left(\bar{\nu}_{\mu} \rightarrow \nu_{\mu}\right) & =\frac{m_{1}^{2}}{E^{2}} \sin ^{2} \theta+\frac{m_{2}^{2}}{E^{2}} \cos ^{2} \theta-P\left(\bar{\nu}_{\mu} \rightarrow \nu_{e}\right),
\end{aligned}
$$




$$
\begin{aligned}
& P\left(\nu_{e} \rightarrow \bar{\nu}_{\mu}\right)=\frac{\sin ^{2} 2 \theta}{4 E^{2}}\left[m_{1}^{2}+m_{2}^{2}-2 m_{1} m_{2} \cos \left(\phi-\frac{\Delta m^{2} L}{2 E}\right)\right]=P\left(\nu_{\mu} \rightarrow \bar{\nu}_{e}\right), \\
& P\left(\bar{\nu}_{e} \rightarrow \nu_{\mu}\right)=\frac{\sin ^{2} 2 \theta}{4 E^{2}}\left[m_{1}^{2}+m_{2}^{2}-2 m_{1} m_{2} \cos \left(\phi+\frac{\Delta m^{2} L}{2 E}\right)\right]=P\left(\bar{\nu}_{\mu} \rightarrow \nu_{e}\right),
\end{aligned}
$$

where $\Delta m^{2}=m_{2}^{2}-m_{1}^{2}$.

It was pointed out in Ref. 25] that the phase $\phi$ can be extracted by measuring the asymmetry

$$
\mathcal{A}=\frac{P\left(\nu_{e} \rightarrow \bar{\nu}_{\mu}\right)-P\left(\bar{\nu}_{e} \rightarrow \nu_{\mu}\right)}{P\left(\nu_{e} \rightarrow \bar{\nu}_{\mu}\right)+P\left(\bar{\nu}_{e} \rightarrow \nu_{\mu}\right)}=\frac{-2 m_{1} m_{2} \sin \phi \sin \left(\frac{\Delta m^{2} L}{2 E}\right)}{m_{1}^{2}+m_{2}^{2}-2 m_{1} m_{2} \cos \phi \cos \left(\frac{\Delta m^{2} L}{2 E}\right)},
$$

in flavor-changing neutrino oscillations.

Neutrinoless double beta $(0 \nu 2 \beta)$ decay experiments are sensitive to the $C P$-even quantity

$$
\left\langle m_{e e}\right\rangle=\left|m_{1} \cos ^{2} \theta+m_{2} \sin ^{2} \theta e^{-i \phi}\right| \equiv\left|\sum_{i} U_{e i}^{2} m_{i}\right|,
$$

which depends on the $C P$-odd phase $\phi$. It is worth noting that the oscillation probability,

$$
P\left(\nu_{e} \rightarrow \bar{\nu}_{e}\right)=\frac{1}{E^{2}}\left|m_{1} \cos ^{2} \theta+m_{2} \sin ^{2} \theta e^{-i\left(\phi-\frac{\Delta m^{2} L}{2 E}\right)}\right|^{2},
$$

is proportional to a related combination with a different phase.

In the case of degenerate neutrinos, $P\left(\nu_{e} \rightarrow \bar{\nu}_{e}\right)$ reduces to

$$
P\left(\nu_{e} \rightarrow \bar{\nu}_{e}\right)=\frac{m^{2}}{E^{2}}\left(\cos ^{4} \theta+\sin ^{4} \theta+2 \sin ^{2} \theta \cos ^{2} \theta \cos \phi\right) \neq \frac{m^{2}}{E^{2}},
$$

which shows that the mixing angle $\theta$ remains physical for degenerate Majorana neutrinos so long as $\phi \neq 0$. One recovers the naive expectation $m^{2} / E^{2}$ only for $\phi=0, \pi$. A non-trivial dependence of flavor-changing neutrino-antineutrino oscillation probabilities on $\theta$ and $\phi$ in the degenerate limit was noticed in Ref. 25]. Eq. (134) shows that flavor-conserving transitions also depend on $\theta$ and $\phi$ in the degenerate limit.

\subsubsection{Low-energy $d \leq 6$-Effective Lagrangian}

Now consider the neutrino Lagrangian in Eq. (47) where the neutrino kinetic energy term is no longer diagonal in flavor. In the physical low-energy basis, the $2 \times 2$ Hermitian matrix $\lambda$ of the light neutrinos depends on 3 real and 1 imaginary parameters

$$
\lambda=\frac{v^{2}}{2} c^{d=6} \equiv\left(\begin{array}{cc}
\lambda_{e e} & \lambda_{e \mu} e^{i \bar{\sigma}} \\
\lambda_{e \mu} e^{-i \bar{\sigma}} & \lambda_{\mu \mu}
\end{array}\right) .
$$

By performing the rephasing of the charged lepton fields given in Eqs. (118) and (119), the physical weak currents can be described in terms of the mixing matrix $U^{\text {eff }}$, Eq. (55),

$$
U^{\mathrm{eff}} \equiv\left(1-\Omega \frac{\lambda}{2} \Omega^{\dagger}\right) U=\left(\begin{array}{cc}
1-\frac{\lambda_{e e}}{2} & -\frac{\lambda_{e \mu}}{2} e^{i \Sigma} \\
-\frac{\lambda_{e \mu}}{2} e^{-i \Sigma} & 1-\frac{\lambda_{\mu \mu}}{2}
\end{array}\right) U
$$


The physical lepton mixing matrix $U^{\text {eff }}$ depends on two phases: the phase $\phi$ of $U$ given in Eq. (40), and the phase

$$
\Sigma \equiv \bar{\sigma}+\left(\omega_{e}-\omega_{\mu}\right)=\bar{\sigma}+\frac{\phi}{2}+\frac{\hat{\theta}_{e}-\hat{\theta}_{\mu}}{2}=\bar{\sigma}+\rho
$$

The low-energy phases $\phi$ and $\Sigma$ (or $\rho$ and $\bar{\sigma}$ in the parameterization in terms of $V^{\text {eff }}$ ) are both invariant under $V_{\ell}=V_{e}$ transformations, as previously stated.

Notice that the phase $\Sigma$ depends on the phases $\hat{\theta}_{\alpha}$, so that these phases cannot be completely removed from the theory when the $d=6$ operator is included.

\subsubsection{Oscillation probabilities for $d \leq 6$-Effective Theory}

The oscillation probabilities derived with the inclusion of only the $d=5$ Majorana mass term are modified by the inclusion of the $d=6$ matrix $\lambda$. The neutrino-neutrino oscillation probabilities are given by

$$
\begin{aligned}
& P\left(\nu_{e} \rightarrow \nu_{e}\right)=\left(1-2 \lambda_{e e}\right)-\left[\left(1-2 \lambda_{e e}\right) \sin ^{2} 2 \theta+2 \lambda_{e \mu} \sin 2 \theta \cos 2 \theta \cos (\Sigma)\right] \sin ^{2}\left(\frac{\Delta m^{2} L}{4 E}\right), \\
& P\left(\nu_{\mu} \rightarrow \nu_{\mu}\right)=\left(1-2 \lambda_{\mu \mu}\right)-\left[\left(1-2 \lambda_{\mu \mu}\right) \sin ^{2} 2 \theta-2 \lambda_{e \mu} \sin 2 \theta \cos 2 \theta \cos (\Sigma)\right] \sin ^{2}\left(\frac{\Delta m^{2} L}{4 E}\right), \\
& P\left(\nu_{e} \rightarrow \nu_{\mu}\right)=\left[1-\left(\lambda_{e e}+\lambda_{\mu \mu}\right)\right] \sin ^{2} 2 \theta \sin ^{2}\left(\frac{\Delta m^{2} L}{4 E}\right)+\lambda_{e \mu} \sin 2 \theta \sin (\Sigma) \sin \left(\frac{\Delta m^{2} L}{2 E}\right), \\
& P\left(\nu_{\mu} \rightarrow \nu_{e}\right)=\left[1-\left(\lambda_{e e}+\lambda_{\mu \mu}\right)\right] \sin ^{2} 2 \theta \sin ^{2}\left(\frac{\Delta m^{2} L}{4 E}\right)-\lambda_{e \mu} \sin 2 \theta \sin (\Sigma) \sin \left(\frac{\Delta m^{2} L}{2 E}\right),
\end{aligned}
$$

where $\Delta m^{2}=m_{2}^{2}-m_{1}^{2}$. With the inclusion of the $d=6$ operator, the sum of the $\nu_{e} \rightarrow \nu_{\alpha}$ oscillation probabilities no longer equals unity:

$$
\begin{aligned}
P\left(\nu_{e} \rightarrow \nu_{e}\right)+P\left(\nu_{e} \rightarrow \nu_{\mu}\right) & =\left(1-2 \lambda_{e e}\right)+\left[\left(\lambda_{e e}-\lambda_{\mu \mu}\right) \sin ^{2} 2 \theta-\lambda_{e \mu} \sin 4 \theta \cos (\Sigma)\right] \sin ^{2}\left(\frac{\Delta m^{2} L}{4 E}\right) \\
& +\lambda_{e \mu} \sin 2 \theta \sin (\Sigma) \sin \left(\frac{\Delta m^{2} L}{2 E}\right) .
\end{aligned}
$$

The main consequence of the inclusion of $d=6$ operator is the appearance of $C P$-violation in conventional neutrino-neutrino oscillations with only 2 neutrino families. The $C P$-odd phase $\Sigma$ leads to the asymmetry

$$
\mathcal{A}=\frac{P\left(\nu_{e} \rightarrow \nu_{\mu}\right)-P\left(\bar{\nu}_{e} \rightarrow \bar{\nu}_{\mu}\right)}{P\left(\nu_{e} \rightarrow \nu_{\mu}\right)+P\left(\bar{\nu}_{e} \rightarrow \bar{\nu}_{\mu}\right)}=4 \lambda_{e \mu} \frac{\sin \Sigma}{\sin 2 \theta} \cot \left(\frac{\Delta m^{2} L}{4 E}\right)
$$

where $C P T$ invariance has been used, i.e. $P\left(\nu_{\mu} \rightarrow \nu_{e}\right)=P\left(\bar{\nu}_{e} \rightarrow \bar{\nu}_{\mu}\right)$.

The neutrino-antineutrino oscillations probabilities and $0 \nu \beta \beta$ decay formulae also are modified when the $d=6$ operator is included. The explicit formulae are lengthy and are relegated to Appendix 3. 


\subsection{Connection between High- and Low-Energy Parameters}

We can now apply the formulae obtained in Sect. 4 to the case of two heavy and two light neutrinos.

In the physical low-energy basis, the $2 \times 2$ Hermitian matrix

$$
\lambda=\left(\begin{array}{cc}
\lambda_{e e} & \lambda_{e \mu} e^{i \bar{\sigma}} \\
\lambda_{e \mu} e^{-i \bar{\sigma}} & \lambda_{\mu \mu}
\end{array}\right)
$$

is diagonalized by the $2 \times 2$ unitary matrix $W$ :

$$
\lambda=W \lambda_{\text {diag, real }} W^{\dagger}=W\left(\begin{array}{cc}
\lambda_{1} & 0 \\
0 & \lambda_{2}
\end{array}\right) W^{\dagger},
$$

where $W$ is parameterized by 1 mixing angle $\theta^{\prime}$ and 1 phase $\bar{\sigma}$,

$$
W=\left(\begin{array}{cc}
\cos \theta^{\prime} & \sin \theta^{\prime} e^{-i \bar{\sigma}} \\
-\sin \theta^{\prime} e^{i \bar{\sigma}} & \cos \theta^{\prime}
\end{array}\right) .
$$

The matrix $V_{N}$ is the unitary matrix which diagonalizes the heavy Majorana neutrino mass matrix $M$,

$$
M_{\text {diag,real }} \equiv V_{N}^{*} M V_{N}^{\dagger},
$$

where $M$ is given in terms of the low-energy parameters by

$$
M \equiv-\left(\sqrt{\lambda_{\text {diag, real }}}\right)^{-1} W^{\dagger} V^{\dagger} m_{\text {diag, real }} V^{*} W^{*}\left(\sqrt{\lambda_{\text {diag, real }}}\right)^{-1},
$$

where $V$ is given by Eq. (115). Thus, $M$ depends on the low-energy phases $\hat{\theta}_{e}, \hat{\theta}_{\mu}, \phi$ and $\bar{\sigma}$, the eigenvalues of the matrices $m$ and $\lambda$, and the mixing angles $\theta$ and $\theta^{\prime}$. $V_{N}$ can be determined as a function of these low-energy parameters as well.

It is useful to rewrite the above in terms of the physical lepton mixing matrix $U$ defined by Eq. (40). Define the matrix

$$
\tilde{W} \equiv \Omega W \Omega^{\dagger}=\left(\begin{array}{cc}
\cos \theta^{\prime} & \sin \theta^{\prime} e^{-i \Sigma} \\
-\sin \theta^{\prime} e^{i \Sigma} & \cos \theta^{\prime}
\end{array}\right)
$$

which diagonalizes

$$
\tilde{\lambda} \equiv \Omega \lambda \Omega^{\dagger}=\left(\begin{array}{cc}
\lambda_{e e} & \lambda_{e \mu} e^{i \Sigma} \\
\lambda_{e \mu} e^{-i \Sigma} & \lambda_{\mu \mu}
\end{array}\right)
$$

Eq. (144) can be rewritten as

$$
M_{\text {diag, real }}=\tilde{V}_{N}^{*} \tilde{M} \tilde{V}_{N}^{\dagger}
$$

where

$$
\tilde{V}_{N} \equiv V_{N} \Omega^{T}
$$


is the unitary matrix which diagonalizes

$$
\tilde{M} \equiv \Omega M \Omega^{T}=-\left(\sqrt{\lambda_{\text {diag, real }}}\right)^{-1} \tilde{W}^{\dagger} U m_{\text {diag, real }} U^{T} \tilde{W}^{*}\left(\sqrt{\lambda_{\text {diag, real }}}\right)^{-1} .
$$

Notice that Eq. (150) implies that the imaginary part of $\tilde{M}$ depends on only the two physical phases $\phi$ and $\Sigma$. The explicit formulae for $\tilde{M}$ are given in Appendix 4 .

Comparison of Eqs. (145) and (150) with Eq. (79) gives

$$
L=V^{*} W^{*}\left(\sqrt{\lambda_{\text {diag, real }}}\right)^{-1} V_{N}^{\dagger}=U^{T} \tilde{W}^{*}\left(\sqrt{\lambda_{\text {diag, real }}}\right)^{-1} \tilde{V}_{N}^{\dagger} .
$$

The matrix $L$ encoding the phase information relevant for leptogenesis is seen to depend on the phases $\phi$ and $\Sigma$.

The combination $Y_{\nu}^{\dagger} Y_{\nu}$ relevant for leptogenesis is given by Eq. (80) with Eq. (151) substituted for $L$,

$$
Y_{\nu}^{\dagger} Y_{\nu}=\tilde{V}_{N}\left(\sqrt{\lambda_{\text {diag, real }}}\right)^{-1} \tilde{W}^{*} U^{*} m_{\text {diag, real }}^{2} U^{T} \tilde{W}^{*}\left(\sqrt{\lambda_{\text {diag, real }}}\right)^{-1} \tilde{V}_{N}^{\dagger} .
$$

The Majorana phases of the light neutrinos appearing in $D_{M a j}$ cancel in the combination $U^{*} m_{\text {diag, real }}^{2} U^{T}$. Thus, any dependence of the leptogenesis asymmetry on the low-energy Majorana phases remains only through the $\tilde{V}_{N}$ matrix.

\section{Conclusions}

The low-energy $d \leq 6$-effective Lagrangian of the seesaw model is equal to the SM Lagrangian plus two higher-dimension operators: a $d=5$ operator, the well-known operator responsible for light Majorana neutrino masses, and a $d=6$ operator. We have determined the connection between the high-energy Lagrangian of the seesaw model and the low-energy effective theory, when the number of heavy neutrinos is less than or equal to the number of light lepton generations.

We have used the chiral symmetries of the seesaw Lagrangian and the effective theory to define physical high-energy and low-energy bases which contain no unphysical parameters. For practical purposes, a standard high-energy basis has been defined. It does not exactly correspond to the usual physical low-energy basis, and the connection between the two has been discussed in detail.

We have illustrated how the measurement of low-energy neutrino-neutrino and neutrinoantineutrino transitions would allow a priori the determination of all the matrix elements of the $d=5$ and $d=6$ operator coefficients, and, thus, all the parameters of the highenergy seesaw Lagrangian. In particular, we have found a non-vanishing $C P$-asymmetry in neutrino-neutrino oscillations, which at leading order is sensitive to the $C P$-phases of the $d=6$ operator coefficient, and is non-vanishing even in the case of only two light neutrino species.

Although in practice the experimental determination of the $d=6$ operator is out of reach for natural values of the seesaw scale, the analysis here has strong implications for the explanation of the matter-antimatter asymmetry of the universe through leptogenesis. 
The latter is shown to require both non-degenerate light neutrino masses and non-degenerate eigenvalues of the $d=6$ operator. When both of these degeneracies simultaneously occur, it is shown that the heavy neutrinos become mass degenerate and there is no leptogenesis asymmetry.

Finally, the general results have been illustrated in full detail for the case of two light and two heavy neutrino generations.

\section{Acknowledgments}

We are indebted to Pilar Hernandez for discussions. A.B and M.B.G were partially supported by CICYT FPA2000-0980 project. A.B acknowledges MECD for financial support by FPU grant AP2001-0521. E.J. was supported in part by the Department of Energy under grant DOE-FG03-97ER40546.

\section{Appendix 1}

In a general basis, the complex symmetric $n^{\prime} \times n^{\prime}$ matrix $M$ contains $n^{\prime}\left(n^{\prime}+1\right) / 2$ real and $n^{\prime}\left(n^{\prime}+1\right) / 2$ imaginary parameters. Consider a basis in which $M$ is diagonal and complex, rather than diagonal and real. The transformation

$$
M \rightarrow V_{N}^{*} M V_{N}^{\dagger}=M_{\text {diag }}=\operatorname{diag}\left(\left|M_{1}\right| e^{i \theta_{1}},\left|M_{2}\right| e^{i \theta_{2}}, \cdots,\left|M_{n^{\prime}}\right| e^{i \theta_{n^{\prime}}}\right),
$$

determines only $n^{\prime}\left(n^{\prime}-1\right) / 2$ real and $n^{\prime}\left(n^{\prime}-1\right) / 2$ imaginary parameters of the $n^{\prime} \times n^{\prime}$ unitary matrix $V_{N}$, which contains $n^{\prime}\left(n^{\prime}-1\right) / 2$ real and $n^{\prime}\left(n^{\prime}+1\right) / 2$ imaginary parameters. $M$ now depends on $n^{\prime}$ real and $n^{\prime}$ imaginary parameters. $n^{\prime}$ imaginary parameters of $V_{N}$ are not determined since $M_{\text {diag }}$ remains diagonal under a transformation

$$
V_{N}^{\dagger}=\operatorname{diag}\left(e^{i \beta_{1}}, e^{i \beta_{2}}, \cdots, e^{i \beta_{n^{\prime}}}\right)
$$

which depends on $n^{\prime}$ arbitrary phases $\beta_{i}$. This rephasing leaves the $n^{\prime}$ real parameters $\left|M_{i}\right|$ invariant, but affects the heavy Majorana phases,

$$
\theta_{i} \rightarrow \theta_{i}+2 \beta_{i}
$$

as well as the phases $\phi_{\alpha j}^{\prime}$ of the matrix

$$
\left(Y_{\nu}^{\prime}\right)_{\alpha j} \equiv u_{\alpha j} e^{i \phi_{\alpha j}^{\prime}}
$$

Under $V_{\ell}=V_{e}$ and $V_{N}$ rephasings, $Y_{\nu}^{\prime}$ transforms as

$$
Y_{\nu}^{\prime} \rightarrow V_{\ell} Y_{\nu}^{\prime} V_{N}^{\dagger}
$$

where $V_{\ell}$ and $V_{N}^{\dagger}$ are given by Eqs. (12) and (154), respectively, so that the phases $\phi_{\alpha j}^{\prime} \rightarrow$ $\phi_{\alpha j}^{\prime}+\zeta_{\alpha}+\beta_{j}$ under the rephasing. 
The heavy Majorana mass eigenstate neutrinos in this alternative basis are defined by

$$
N_{i}=e^{i \theta_{i} / 2} N_{R i}+e^{-i \theta_{i} / 2} N_{R i}{ }^{c} \equiv \sqrt{\eta_{i}} N_{R i}+\sqrt{\eta_{i}{ }^{*}} N_{R i}{ }^{c},
$$

and continue to satisfy the condition $N_{i}{ }^{c}=N_{i}$.

The above basis is related to the standard high-energy basis in the text by a rephasing with $\beta_{i}=-\theta_{i} / 2$ under which $M$ becomes diagonal and real, and $Y_{\nu}^{\prime} \rightarrow Y_{\nu}=Y_{\nu}^{\prime} \sqrt{\eta^{*}}$, with phases $\phi_{\alpha j} \equiv\left(\phi_{\alpha j}^{\prime}-\theta_{j} / 2\right)$. The phases $\phi_{\alpha j}$, which are used in the standard high-energy basis, are invariant under $\beta_{i}$ rephasings.

The coefficients of the higher-dimensional operators of the low-energy effective theory do not depend on $\beta_{i}$ rephasings, so the low-energy coefficients computed in a general basis are equal to those computed in the standard high-energy basis.

\section{Appendix 2}

Consider the low-energy theory including only the effects of the $d=5$ operator, for the case $n=n^{\prime}=2$ generations. In the body of the paper, we expressed the flavor eigenstate parameters in terms of the mass eigenstate parameters. Here the inverse procedure [23] is shown in detail: the mass eigenstate parameters are given in terms of the flavor eigenstate parameters.

The light neutrino mass matrix

$$
\left(\begin{array}{cc}
m_{e e} e^{i \gamma_{e}} & m_{e \mu} e^{i \gamma_{e \mu}} \\
m_{e \mu} e^{i \gamma_{e \mu}} & m_{\mu \mu} e^{i \gamma_{\mu}}
\end{array}\right)
$$

is brought to diagonal and real form with eigenvalues $m_{1}$ and $m_{2}$ by the matrix $V^{\prime}$

$$
\left(\begin{array}{cc}
m_{1} & 0 \\
0 & m_{2}
\end{array}\right)=V^{\prime *}\left(\begin{array}{cc}
m_{e e} e^{i \gamma_{e}} & m_{e \mu} e^{i \gamma_{e \mu}} \\
m_{e \mu} e^{i \gamma_{e \mu}} & m_{\mu \mu} e^{i \gamma_{\mu}}
\end{array}\right) V^{\prime \dagger}
$$

where

$$
V^{\prime \dagger}=\left(\begin{array}{cc}
e^{-i \gamma_{e} / 2} & 0 \\
0 & e^{-i \gamma_{\mu} / 2}
\end{array}\right) V^{\dagger}
$$

and $V$ is given by Eqs. (115) and (116). Eq. (160) can be rewritten as Eq. (114) in terms of the basis-independent phase $\bar{\gamma}$ defined in Eq. (108). Explicitly,

$$
\begin{aligned}
\left(\begin{array}{cc}
m_{1} & 0 \\
0 & m_{2}
\end{array}\right) & =\left(\begin{array}{cc}
e^{-i \hat{\theta}_{e} / 2} & 0 \\
0 & e^{-i \hat{\theta}_{\mu} / 2}
\end{array}\right)\left(\begin{array}{cc}
\cos \theta & -\sin \theta e^{i \rho} \\
\sin \theta e^{-i \rho} & \cos \theta
\end{array}\right) \\
& \times\left(\begin{array}{cc}
m_{e e} & m_{e \mu} e^{i \bar{\gamma}} \\
m_{e \mu} e^{i \bar{\gamma}} & m_{\mu \mu}
\end{array}\right) \\
& \times\left(\begin{array}{cc}
\cos \theta & \sin \theta e^{-i \rho} \\
-\sin \theta e^{i \rho} & \cos \theta
\end{array}\right)\left(\begin{array}{cc}
e^{-i \hat{\theta}_{e} / 2} & 0 \\
0 & e^{-i \hat{\theta}_{\mu} / 2}
\end{array}\right),
\end{aligned}
$$

where the parameterization of $V$ in Eq. (116) has been used. 
The correspondence between the results of Ref. [23] and Eqs. (122)-(123) then is obtained immediately by bringing the light Majorana neutrino mass matrix to diagonal and complex form with the rephasing

$$
V_{\ell}=\left(\begin{array}{cc}
e^{-i \hat{\theta}_{e} / 2} & 0 \\
0 & e^{-i \hat{\theta}_{\mu} / 2}
\end{array}\right)
$$

The diagonal entries in Eq. (162) yield

$$
\begin{aligned}
& m_{1} e^{i \hat{\theta}_{e}}=m_{e e} \cos ^{2} \theta-m_{e \mu} e^{i(\bar{\gamma}+\rho)} \sin 2 \theta+m_{\mu \mu} e^{i 2 \rho} \sin ^{2} \theta \\
& m_{2} e^{i \hat{\theta}_{\mu}}=m_{e e} e^{-i 2 \rho} \sin ^{2} \theta+m_{e \mu} e^{i(\bar{\gamma}-\rho)} \sin 2 \theta+m_{\mu \mu} \cos ^{2} \theta .
\end{aligned}
$$

The condition that the off-diagonal matrix elements vanish is [23]:

$$
\left(m_{\mu \mu} e^{i \rho}-m_{e e} e^{-i \rho}\right) \sin 2 \theta=2 m_{e \mu} e^{i \bar{\gamma}} \cos 2 \theta .
$$

Taking the real and the imaginary parts of Eq. (164) and Eq. (165), we obtain six conditions on the parameters

$$
\begin{aligned}
m_{1} \cos \hat{\theta}_{e} & =m_{e e} \cos ^{2} \theta-m_{e \mu} \cos (\bar{\gamma}+\rho) \sin 2 \theta+m_{\mu \mu} \cos 2 \rho \sin ^{2} \theta, \\
m_{2} \cos \hat{\theta}_{\mu} & =m_{e e} \cos 2 \rho \sin ^{2} \theta+m_{e \mu} \cos (\bar{\gamma}-\rho) \sin 2 \theta+m_{\mu \mu} \cos ^{2} \theta, \\
m_{1} \sin \hat{\theta}_{e} & =-m_{e \mu} \sin (\bar{\gamma}+\rho) \sin 2 \theta+m_{\mu \mu} \sin 2 \rho \sin ^{2} \theta \\
m_{2} \sin \hat{\theta}_{\mu} & =-m_{e e} \sin 2 \rho \sin ^{2} \theta+m_{e \mu} \sin (\bar{\gamma}-\rho), \sin 2 \theta \\
0 & =-\left(m_{\mu \mu}-m_{e e}\right) \cos \rho \sin 2 \theta+2 m_{e \mu} \cos \bar{\gamma} \cos 2 \theta \\
0 & =-\left(m_{\mu \mu}+m_{e e}\right) \sin \rho \sin 2 \theta+2 m_{e \mu} \sin \bar{\gamma} \cos 2 \theta
\end{aligned}
$$

We can analyze these equations in different cases, three of them considered in Ref [23]:

- Case 1: $\bar{\gamma}=0$ with $m_{e e} \neq m_{\mu \mu}$.

In this case, Eq. (171) implies that $\rho=0, \pi$ and Eq. (170) yields

$$
\tan (2 \theta)=\frac{2 m_{e \mu}}{m_{\mu \mu}-m_{e e}} .
$$

Let's choose, for instance, $\rho=0$. Eq. (168) and Eq. (169) imply that $\hat{\theta}_{e}=\hat{\theta}_{\mu}=0, \pi$, which means no $C P$ violation. The same holds for $\rho=\pi$.

For instance, for $\rho=\hat{\theta}_{e}=\hat{\theta}_{\mu}=0$, the mass eigenvalues read:

$$
\begin{aligned}
& m_{1}=m_{e e} \cos ^{2} \theta+m_{\mu \mu} \sin ^{2} \theta-2 m_{e \mu} \sin \theta \cos \theta, \\
& m_{2}=m_{e e} \sin ^{2} \theta+m_{\mu \mu} \cos ^{2} \theta+2 m_{e \mu} \sin \theta \cos \theta,
\end{aligned}
$$

and the mixing matrix appearing in Eq. (40) is

$$
U=\left(\begin{array}{cc}
\cos \theta & \sin \theta \\
-\sin \theta & \cos \theta
\end{array}\right)
$$


- Case 2a: $\bar{\gamma} \neq 0$ with $m_{e e}=m_{\mu \mu}$.

Now, Eq. (170) yields $\theta=\pi / 4$. Substituting these values in Eq. (171), one finds $\rho=0, \pi$. Substituting this set of values in Eq. (168) and Eq. (169) yields

$$
\begin{aligned}
& m_{1} \sin \hat{\theta}_{e}=\mp m_{e \mu} \sin \bar{\gamma}, \\
& m_{2} \sin \hat{\theta}_{\mu}= \pm m_{e \mu} \sin \bar{\gamma} .
\end{aligned}
$$

The real parts in Eq. (166) and Eq. (167) result in the equations

$$
\begin{aligned}
& m_{1} \cos \hat{\theta}_{e}=m_{e e} \mp m_{e \mu} \cos \bar{\gamma} \\
& m_{2} \cos \hat{\theta}_{\mu}=m_{e e} \pm m_{e \mu} \cos \bar{\gamma}
\end{aligned}
$$

Since $\bar{\gamma} \neq 0$, the $C P$ parities $\hat{\theta}_{e}, \hat{\theta}_{\mu} \neq 0, \pi$.

Finally, the mixing matrix is given by

$$
U=\left(\begin{array}{cc}
\frac{1}{\sqrt{2}} & \frac{1}{\sqrt{2}} \\
-\frac{1}{\sqrt{2}} & \frac{1}{\sqrt{2}}
\end{array}\right)\left(\begin{array}{cc} 
\pm e^{-i \frac{\hat{\theta}_{e}-\hat{\theta}_{\mu}}{2}} & 0 \\
0 & 1
\end{array}\right) .
$$

- Case 2b: $\bar{\gamma} \neq 0$ with $m_{e e}=-m_{\mu \mu}$.

Now, Eq. (170) yields $\theta=\pi / 4$. Substituting these values in Eq. (171), one finds $\rho= \pm \pi / 2$. Eq. (168) and Eq. (169) reduce to

$$
\begin{aligned}
& m_{1} \sin \hat{\theta}_{e}=\mp m_{e \mu} \cos \bar{\gamma} \\
& m_{2} \sin \hat{\theta}_{\mu}=\mp m_{e \mu} \cos \bar{\gamma} .
\end{aligned}
$$

The real parts in Eq. (166) and Eq. (167) are

$$
\begin{aligned}
& m_{1} \cos \hat{\theta}_{e}=m_{e e} \pm m_{e \mu} \sin \bar{\gamma} \\
& m_{2} \cos \hat{\theta}_{\mu}=-m_{e e} \pm m_{e \mu} \sin \bar{\gamma}
\end{aligned}
$$

and the mixing matrix that governs the $\mathrm{CC}$ current is

$$
U=\left(\begin{array}{cc}
\frac{1}{\sqrt{2}} & \frac{1}{\sqrt{2}} \\
-\frac{1}{\sqrt{2}} & \frac{1}{\sqrt{2}}
\end{array}\right)\left(\begin{array}{cc}
e^{i\left( \pm \frac{\pi}{2}-\frac{\hat{\theta}_{e}-\hat{\theta}_{\mu}}{2}\right)} & 0 \\
0 & 1
\end{array}\right) \text {. }
$$

Notice that the $C P$ parities can only be $\bar{\theta}_{e}=\bar{\theta}_{\mu}=0, \pi$ for the special case $\bar{\gamma}=\pi / 2$.

- Case 3: $\bar{\gamma} \neq 0$ with $m_{e e} \neq \pm m_{\mu \mu}$. In this case Eq. (170) and Eq. (171) can be rewritten as

$$
\begin{aligned}
\tan \rho & =\frac{m_{\mu \mu}-m_{e e}}{m_{e e}+m_{\mu \mu}} \tan \bar{\gamma}, \\
\tan 2 \theta & =\frac{2 m_{e \mu}}{m_{\mu \mu}-m_{e e}} \frac{\cos \bar{\gamma}}{\cos \rho}=\frac{2 m_{e \mu}}{m_{e e}+m_{\mu \mu}} \frac{\sin \bar{\gamma}}{\sin \rho},
\end{aligned}
$$

which, together with Eq. (166)-Eq. (169), allow all physical low-energy parameters to be determined.

The physical mixing matrix is

$$
U=\left(\begin{array}{cc}
\cos \theta & \sin \theta \\
-\sin \theta & \cos \theta
\end{array}\right)\left(\begin{array}{cc}
e^{i \phi / 2} & 0 \\
0 & 1
\end{array}\right)
$$

with $\phi$ given by Eq. (117). 


\section{Appendix 3}

For two generations, the neutrino-antineutrino oscillation probabilities including the $d=5$ and the dominant $d=6$ coefficient are given by

$$
\begin{aligned}
P\left(\nu_{e} \rightarrow \bar{\nu}_{e}\right) & =\left(1-2 \lambda_{e e}\right) \frac{1}{E^{2}}\left\{m_{1}^{2} \cos ^{4} \theta+m_{2}^{2} \sin ^{4} \theta+m_{1} m_{2} \frac{\sin 2 \theta}{2} \cos \left(\phi-\frac{\Delta m^{2} L}{2 E}\right)\right\} \\
& +\lambda_{e \mu} \frac{\sin 2 \theta}{E^{2}}\left\{\left(m_{1}^{2} \cos ^{2} \theta-m_{2}^{2} \sin ^{2} \theta\right) \cos \Sigma\right. \\
& \left.+m_{1} m_{2}\left[\sin ^{2} \theta \cos \left(\Sigma+\phi-\frac{\Delta m^{2} L}{2 E}\right)-\cos ^{2} \theta \cos \left(\Sigma-\phi+\frac{\Delta m^{2} L}{2 E}\right)\right]\right\} \\
P\left(\nu_{\mu} \rightarrow \bar{\nu}_{\mu}\right) & =\left(1-2 \lambda_{\mu \mu}\right) \frac{1}{E^{2}}\left\{m_{1}^{2} \sin ^{4} \theta+m_{2}^{2} \cos ^{4} \theta+m_{1} m_{2} \frac{\sin 2 \theta}{2} \cos \left(\phi-\frac{\Delta m^{2} L}{2 E}\right)\right\} \\
& +\lambda_{e \mu} \frac{\sin 2 \theta}{E^{2}}\left\{\left(m_{1}^{2} \sin ^{2} \theta-m_{2}^{2} \cos ^{2} \theta\right) \cos \Sigma\right. \\
& \left.+m_{1} m_{2}\left[\cos ^{2} \theta \cos \left(\Sigma-\phi+\frac{\Delta m^{2} L}{2 E}\right)-\sin ^{2} \theta \cos \left(\Sigma+\phi-\frac{\Delta m^{2} L}{2 E}\right)\right]\right\} \\
P\left(\bar{\nu}_{\mu} \rightarrow \nu_{\mu}\right) & =P\left(\nu_{\mu} \rightarrow \bar{\nu}_{\mu}\right)[\phi \rightarrow-\phi] . \\
P\left(\bar{\nu}_{e} \rightarrow \nu_{e}\right) & =P\left(\nu_{e} \rightarrow \bar{\nu}_{e}\right)[\phi \rightarrow-\phi], \\
P\left(\nu_{e} \rightarrow \bar{\nu}_{\mu}\right) & \left.=\left(1-\lambda_{e e}-\lambda_{\mu \mu}\right) \frac{\sin ^{2} 2 \theta}{4 E^{2}}\left[m_{1}^{2}+m_{2}^{2}-2 m_{1} m_{2} \cos \left(\phi-\frac{\Delta m^{2} L}{2 E}\right)\right]\right] \\
& -\lambda_{e \mu} \frac{\sin 2 \theta}{2 E^{2}} \Delta m^{2} \cos \Sigma=P\left(\nu_{\mu} \rightarrow \bar{\nu}_{e}\right), \\
& P\left(\nu_{e} \rightarrow \bar{\nu}_{\mu}\right)[\phi \rightarrow-\phi]=P\left(\bar{\nu}_{\mu} \rightarrow \nu_{e}\right), \\
& \\
P & \\
P &
\end{aligned}
$$

The $C P$-even quantity probed in $0 \nu \beta \beta$-decay becomes

$$
\begin{aligned}
\left\langle m_{e e}\right\rangle & \equiv\left|m_{1}\left(U_{e 1}^{\mathrm{eff}}\right)^{2}+m_{2}\left(U_{e 2}^{\mathrm{eff}}\right)^{2}\right| \\
& =\left|m_{1} \cos ^{2} \theta\left[1-\lambda_{e e}+\lambda_{e \mu} \tan \theta e^{i \Sigma}\right]+m_{2} \sin ^{2} \theta\left[1-\lambda_{e e}-\lambda_{e \mu} \cot \theta e^{i \Sigma}\right] e^{-i \phi}\right| .
\end{aligned}
$$

Comparison of this quantity with the oscillation probability

$$
P\left(\nu_{e} \rightarrow \bar{\nu}_{e}\right)=\frac{1}{E^{2}}\left|m_{1}\left(U_{e 1}^{\text {eff }}\right)^{2}+m_{2}\left(U_{e 2}^{\text {eff }}\right)^{2} e^{i \frac{\Delta m^{2} L}{2 E}}\right|^{2}
$$




$$
\begin{aligned}
=\frac{1}{E^{2}} \mid & m_{1} \cos ^{2} \theta\left[1-\lambda_{e e}+\lambda_{e \mu} \tan \theta e^{i \Sigma}\right] \\
& +\left.m_{2} \sin ^{2} \theta\left[1-\lambda_{e e}-\lambda_{e \mu} \cot \theta e^{i \Sigma}\right] e^{-i\left(\phi-\frac{\Delta m^{2} L}{2 E}\right)}\right|^{2},
\end{aligned}
$$

shows that the simple relationship between these quantities which was apparent when only the $d=5$ operator is included (see Eqs. (132) and (133) ) continues to hold when the $d=6$ operator is included.

\section{Appendix 4}

In the case of two generations, the matrix $\tilde{M}$, defined in Eq. (150), has matrix elements

$$
\tilde{M} \equiv\left(\begin{array}{cc}
\tilde{M}_{11} & \tilde{M}_{12} \\
\tilde{M}_{12} & \tilde{M}_{22}
\end{array}\right)=\left(\begin{array}{cc}
\left|\tilde{M}_{11}\right| e^{i \Gamma_{11}} & \left|\tilde{M}_{12}\right| e^{i \Gamma_{12}} \\
\left|\tilde{M}_{12}\right| e^{i \Gamma_{12}} & \left|\tilde{M}_{22}\right| e^{i \Gamma_{22}}
\end{array}\right)
$$

where the magnitudes $\left|\tilde{M}_{i j}\right|$ and phases $\left|\Gamma_{i j}\right|=\arg \left\{\tilde{M}_{i j}\right\}$ depend on the low-energy phases $\phi$ and $\Sigma$, the eigenvalues of $m$ and $\lambda$, and the mixing angles $\theta$ and $\theta^{\prime}$ :

$$
\begin{array}{rrr}
\tilde{M}_{11}= & -\frac{e^{-i \Sigma}}{\lambda_{1}}\left[m_{1} e^{i \phi}\left(s^{2} \theta^{\prime} s^{2} \theta e^{-i \Sigma}+c^{2} \theta^{\prime} c^{2} \theta e^{i \Sigma}+\frac{1}{2} s 2 \theta^{\prime} s 2 \theta\right)\right. \\
& \left.+m_{2}\left(s^{2} \theta^{\prime} c^{2} \theta e^{-i \Sigma}+c^{2} \theta^{\prime} s^{2} \theta e^{i \Sigma}-\frac{1}{2} s 2 \theta^{\prime} s 2 \theta\right)\right], \\
\tilde{M}_{22}= & -\frac{e^{i \Sigma}}{\lambda_{2}}\left[m_{1} e^{i \phi}\left(s^{2} \theta^{\prime} c^{2} \theta e^{i \Sigma}+c^{2} \theta^{\prime} s^{2} \theta e^{-i \Sigma}-\frac{1}{2} s 2 \theta^{\prime} s 2 \theta\right)\right. \\
& \left.+m_{2}\left(s^{2} \theta^{\prime} s^{2} \theta e^{i \Sigma}+c^{2} \theta^{\prime} c^{2} \theta e^{-i \Sigma}+\frac{1}{2} s 2 \theta^{\prime} s 2 \theta\right)\right], \\
\tilde{M}_{12}= & -\frac{1}{2 \sqrt{\lambda_{1} \lambda_{2}}}\left[m_{1} e^{i \phi}\left(s 2 \theta^{\prime}\left(c^{2} \theta e^{i \Sigma}-s^{2} \theta e^{-i \Sigma}\right)-c 2 \theta^{\prime} s 2 \theta\right)\right. \\
& \left.+m_{2}\left(s^{2} \theta^{\prime}\left(s^{2} \theta e^{i \Sigma}-c^{2} \theta e^{-i \Sigma}\right)+\frac{1}{2} c 2 \theta^{\prime} s 2 \theta\right)\right],
\end{array}
$$

where $s \equiv \sin$ and $c \equiv \cos$.

$\tilde{V}_{N}$ is parametrized in analogy to the diagonalizing matrix $V^{\prime}$ of the light Majorana neutrino mass matrix, Eqs. (160) and (115),

$$
V_{N}^{\dagger}=\left(\begin{array}{cc}
e^{-i \Gamma_{1} / 2} & 0 \\
0 & e^{-i \Gamma_{2} / 2}
\end{array}\right)\left(\begin{array}{cc}
\cos \Theta & \sin \Theta e^{-i \varrho} \\
-\sin \Theta e^{i \varrho} & \cos \Theta
\end{array}\right)\left(\begin{array}{cc}
e^{-i \theta_{1} / 2} & 0 \\
0 & e^{-i \theta_{2} / 2}
\end{array}\right),
$$

where $\Gamma_{1} \equiv \Gamma_{11}$ and $\Gamma_{2} \equiv \Gamma_{22}$. Eq. (13) then becomes

$$
\begin{aligned}
\left(\begin{array}{cc}
\left|M_{1}\right| & 0 \\
0 & \left|M_{2}\right|
\end{array}\right) & =\left(\begin{array}{cc}
e^{-i \theta_{1} / 2} & 0 \\
0 & e^{-i \theta_{2} / 2}
\end{array}\right)\left(\begin{array}{cc}
\cos \Theta & -\sin \Theta e^{i \varrho} \\
\sin \Theta e^{-i \varrho} & \cos \Theta
\end{array}\right) \\
& \times\left(\begin{array}{cc}
\left|\tilde{M}_{11}^{\prime}\right| & \left|\tilde{M}_{12}^{\prime}\right| e^{i \bar{\Gamma}} \\
\left|\tilde{M}_{12}^{\prime}\right| e^{i \bar{\Gamma}} & \left|\tilde{M}_{22}^{\prime}\right|
\end{array}\right) \\
& \times\left(\begin{array}{cc}
\cos \Theta & \sin \Theta e^{-i \varrho} \\
-\sin \Theta e^{i \varrho} & \cos \Theta
\end{array}\right)\left(\begin{array}{cc}
e^{-i \theta_{1} / 2} & 0 \\
0 & e^{-i \theta_{2} / 2}
\end{array}\right)
\end{aligned}
$$


where

$$
\bar{\Gamma} \equiv \Gamma_{12}-\frac{\Gamma_{1}-\Gamma_{2}}{2}
$$

depends on the low-energy real parameters and phases $\phi$ and $\Sigma$. Notice that this diagonalization is identical to that done for the light Majorana neutrino mass matrix with the replacements

$$
\begin{array}{cc}
m_{i} \rightarrow\left|M_{i}\right|, & m_{\alpha \beta} \rightarrow\left|\tilde{M}_{i j}\right|, \\
\theta & \rightarrow \Theta, \quad \bar{\gamma} \rightarrow \bar{\Gamma}, \\
\rho & \rightarrow \varrho, \quad \hat{\theta}_{\alpha} \rightarrow \theta_{i} .
\end{array}
$$

Following computations analogous to the ones performed in the diagonalization of the light neutrino mass matrix, we can determine the heavy mass eigenvalues as a (complicated) function of the low-energy parameters.

\section{$5 \quad$ Appendix 5}

The Feynman rules of the high-energy seesaw model and the low-energy $d \leq 5$ - and $d \leq 6$ effective theories are given in this appendix.

\subsection{Seesaw model}

\section{Yukawa couplings:}

In the basis in which $M$ is a diagonal real matrix, its mass eigenstates are

$$
N_{i} \equiv N_{R i}+N_{R i}{ }^{c} .
$$

The Feynman rules for the Yukawa couplings of the heavy neutrinos to the light lepton doublets are given by:
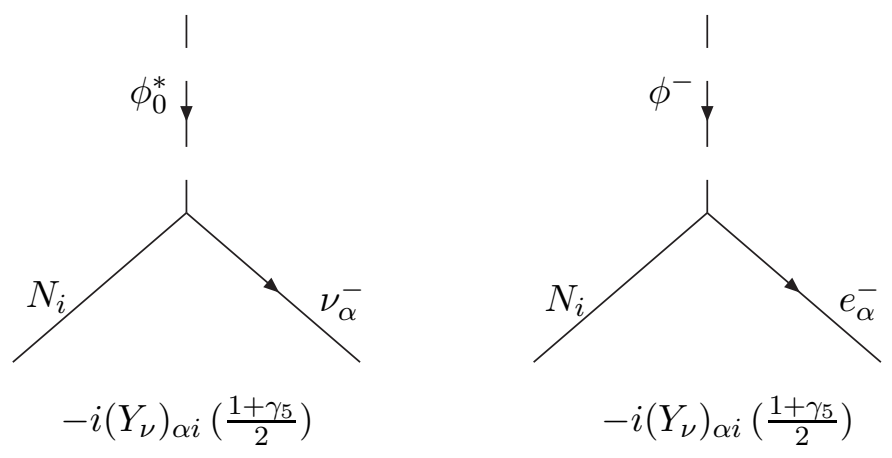

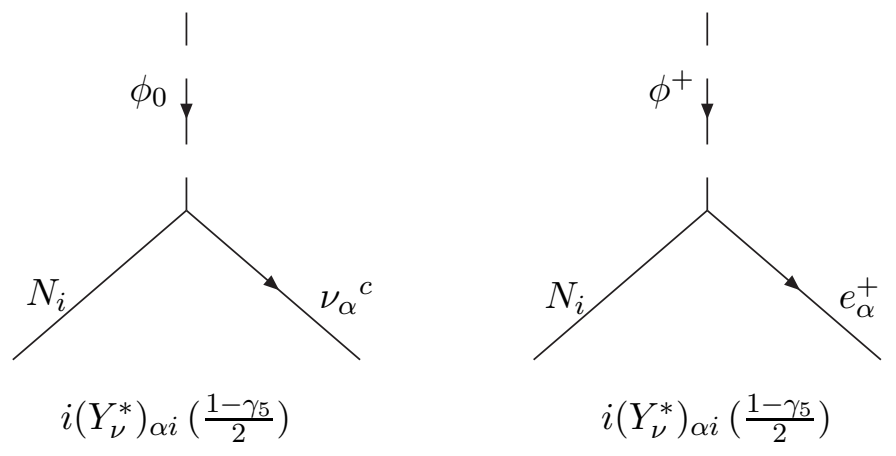

\subsection{Effective theory}

\section{Charged currents: $\mathcal{L}_{S M}+\delta \mathcal{L}^{d=5}$}

In the basis in which $m$ is a diagonal real matrix and the mixing matrix is

$$
U=\left(\begin{array}{cc}
\cos \theta & \sin \theta \\
-\sin \theta & \cos \theta
\end{array}\right)\left(\begin{array}{cc}
e^{i \phi / 2} & 0 \\
0 & 1
\end{array}\right)
$$

the Feynman rules for the $\mathrm{CC}$ are:
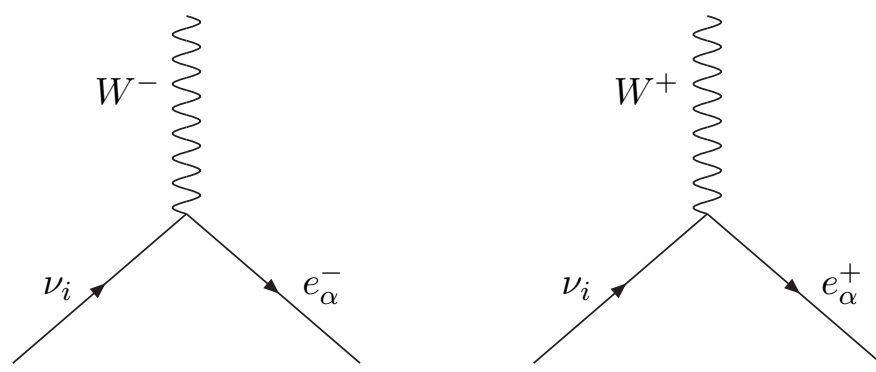

$$
i \frac{g}{2 \sqrt{2}} \gamma_{\mu}\left(\frac{1-\gamma_{5}}{2}\right) U_{\alpha i}
$$
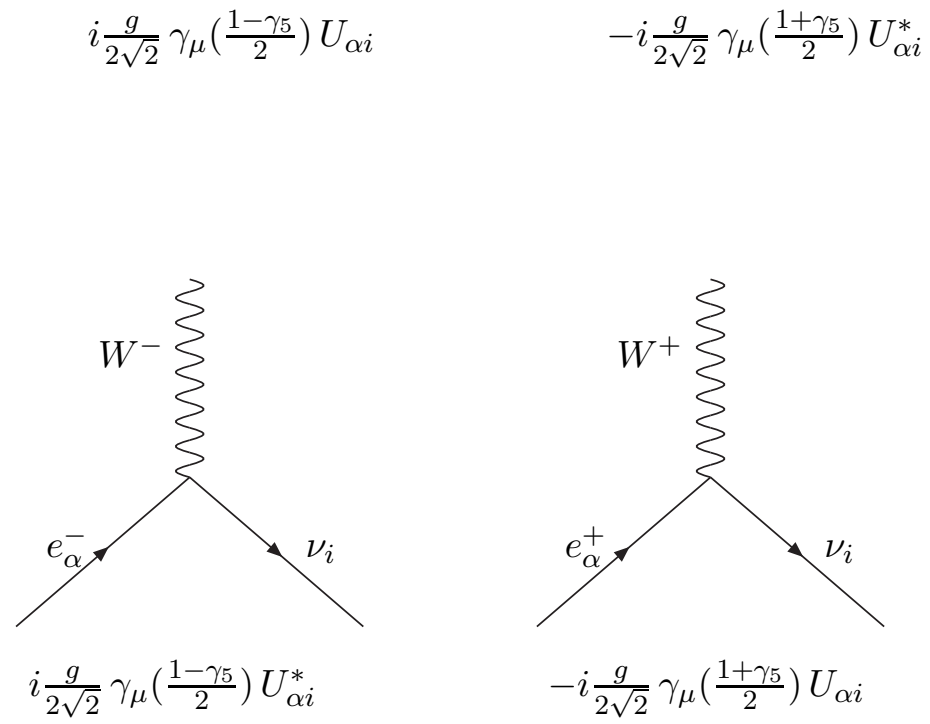
Charged currents: $\mathcal{L}_{S M}+\delta \mathcal{L}^{d=5}+\delta \mathcal{L}^{d=6}$

In the same basis as before, the Feynman rules for the charged currents, including both the $d=5$ and $d=6$ operators, are obtained from the above Feynman rules for the $d \leq 5$-effective theory with the substitution

$$
U \rightarrow U^{\mathrm{eff}} \equiv\left(\begin{array}{cc}
1-\frac{1}{2} \lambda_{e e} & -\frac{1}{2} \lambda_{e \mu} e^{i \Sigma} \\
-\frac{1}{2} \lambda_{e \mu} e^{-i \Sigma} & 1-\frac{1}{2} \lambda_{\mu \mu}
\end{array}\right) U
$$

\section{References}

[1] M. Gell-Mann, P. Ramond and R. Slansky, in Supergravity, edited by P. van Nieuwenhuizen and D. Freedman, (North-Holland, 1979), p. 315; T. Yanagida, in Proceedings of the Workshop on the Unified Theory and the Baryon Number in the Universe, edited by O. Sawada and A. Sugamoto (KEK Report No. 79-18, Tsukuba, 1979), p. 95; R.N. Mohapatra and G. Senjanović, Phys. Rev. Lett. 44 (1980) 912.

[2] M. Fukugita and T. Yanagida, Phys. Lett. 174B (1986) 45.

[3] N. Cabibbo, Phys. Rev. Lett. 10 (1963) 531; M. Kobayashi and T. Maskawa, Prog. Theor. Phys. 49 (1973) 652.

[4] S. M. Bilenky, J. Hosek and S. T. Petcov, Phys. Lett. B 94 (1980) 495; M. Doi, T. Kotani, H. Nishiura, K. Okuda and E. Takasugi, Phys. Lett. B 102 (1981) 323; J. Schechter and J. W. Valle, Phys. Rev. D 25 (1982) 2951.

[5] V. Barger, S. L. Glashow, P. Langacker and D. Marfatia, Phys. Lett. B 540 (2002) 247 arXiv:hep-ph/0205290.

[6] S. Pascoli, S. T. Petcov and W. Rodejohann, Phys. Lett. B 549 (2002) 177 arXiv:hep-ph/0209059.

[7] See, for example, H. Minakata, H. Nunokawa and S. Parker, arXiv:hep-ph/0301210.

[8] See, for example, O. Yasuda, arXiv:hep-ph/0209127, and B. Autin, D. A. Harris, S. F. King, K. S. McFarland and O. Yasuda, arXiv:hep-ph/0302218

[9] A. Broncano, M.B. Gavela and E. Jenkins, Phys. Lett. B 522 (2003) 177 arXiv:hep-ph/0210271.

[10] S. Weinberg, Phys. Rev. Lett. 43 (1979) 1571.

[11] A. Broncano, M. B. Gavela and E. Jenkins, work in progress.

[12] M. Luty, Phys. Rev. D 45 (1992) 455.

[13] M. Flanz, E. A. Paschos and U. Sarkar, Phys. Lett. B 345 (1995) 248 [Erratum-ibid. B 382 (1996) 447] arXiv:hep-ph/9411366; L. Covi, E. Roulet and F. Vissani, Phys. Lett. B 384 (1996) 169 arXiv:hep-ph/9605319; A. Pilaftsis, Phys. Rev. D 56 (1997) 5431 arXiv:hep-ph/9707235|; W. Buchmuller and M. Plumacher, Phys. Lett. B 431 (1998) 354 arXiv:hep-ph/9710460. 
[14] D. N. Spergel et al., arXiv:astro-ph/0302209

[15] W. Buchmuller, P. Di Bari and M. Plumacher, Phys. Lett. B 547 (2002) 128 arXiv:hep-ph/0209301.

[16] B. Pontecorvo, Zh. Eksp. Teor. Fiz.33, 549 (1957) and 34, 247 (1958); Z. Maki, M. Nakagawa and S. Sakata, Prog. Theor. Phys. 28 (1962) 870.

[17] S. Davidson and A. Ibarra, Phys. Lett. B 535 (2002) 25 arXiv:hep-ph/0202239.

[18] J. A. Casas and A. Ibarra, Nucl. Phys. B 618 (2001) 171 arXiv:hep-ph/0103065.

[19] S. Pascoli, S. T. Petcov and W. Rodejohann, arXiv:hep-ph/0302054.

[20] T. Endoh, T. Morozumi, T. Onogi and A. Purwanto, Phys. Rev. D 64 (2001) 013006 [Erratum-ibid. D 64 (2001) 059904] arXiv:hep-ph/0012345; G. C. Branco, T. Morozumi, B. M. Nobre and M. N. Rebelo, Nucl. Phys. B 617 (2001) 475 arXiv:hep-ph/0107164.

[21] F. Borzumati and A. Masiero, Phys. Rev. Lett. 57 (1986) 961.

[22] J. Ellis,J. Hisano,S. Lola and M. Raidal, Nucl. Phys. B 621 (2002) 208. arXiv:hep-ph/0109125; S. Pascoli, S. T. Petcov and C. E. Yaguna, arXiv:hep-ph/0302095.

[23] A. Barroso and J. Maalampi, Phys. Lett. B 132 (1983) 355.

[24] J. Bernabeu and P. Pascual, Nucl. Phys. B 228 (1983) 21; J. Schechter and J. W. Valle, Phys. Rev. D 23 (1981) 1666.

[25] A. de Gouvea, B. Kayser and R. N. Mohapatra, Phys. Rev. D 67 (2003) 053004 arXiv:hep-ph/0211394. 